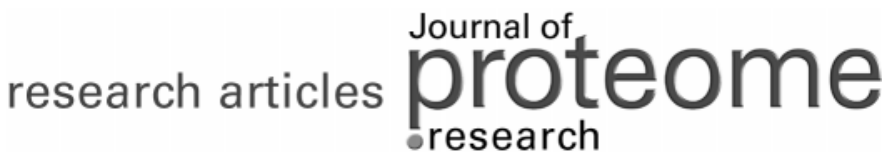

\section{Quantitative Proteomic and Genomic Profiling Reveals Metastasis-Related Protein Expression Patterns in Gastric Cancer Cells}

\author{
Yet-Ran Chen, ${ }^{\dagger}$ Hsueh-Fen Juan, ${ }^{\ddagger}$ Hsuan-Cheng Huang, ${ }^{\circledR}$ Hsin-Hung Huang, ${ }^{\dagger}$ Ya-Jung Lee, ${ }^{\dagger}$ \\ Mei-Yueh Liao," Chien-Wei Tseng, ${ }^{\ddagger}$ Li-Ling Lin, ${ }^{\ddagger}$ Jeou-Yuan Chen, ${ }^{\perp}$ Mei-Jung Wang, ${ }^{\perp}$ \\ Jenn-Han Chen, ${ }^{, 0}$ and Yu-Ju Chen, ${ }^{*,+, \#}$ \\ Institute of Chemistry and Genomic Research Center, Academia Sinica, Taipei, Taiwan, Department of Life \\ Science and Institute of Molecular and Cellular Biology, National Taiwan University, Taipei, Taiwan, Institute \\ of Bioinformatics, National Yang-Ming University, Taipei, Taiwan, Institute of Bioscience and Biotechnology, \\ National Taiwan Ocean University, Keelung, Taiwan, Institute of Biomedical Sciences, Academia Sinica, \\ Taipei, Taiwan, School of Dentistry, National Defense Medical Center, National Defense University, Taipei, \\ Taiwan, and Institute of Molecular Biology, National Chung Hsing University, Taichung, Taiwan
}

Received May 4, 2006

\begin{abstract}
Gastric cancer is a leading cause of death worldwide, and patients have an overall 5-year survival rate of less than $10 \%$. Using quantitative proteomic techniques together with microarray chips, we have established comprehensive proteome and transcriptome profiles of the metastatic gastric cancer TMC-1 cells and the noninvasive gastric cancer SC-M1 cell. Our qualitative protein profiling strategy offers the first comprehensive analysis of the gastric cancer cell proteome, identifying 926 and 909 proteins from SC-M1 and TMC-1 cells, respectively. Cleavable isotope-coded affinity tagging analysis allows quantitation of a total of 559 proteins (with a protein false-positive rate of $<0.005$ ), and 240 proteins were differentially expressed (>1.3-fold) between the SC-M1 and TMC-1 cells. We identified numerous proteins not previously associated with gastric cancer. Notably, a large subset of differentially expressed proteins was associated with tumor metastasis, including proteins functioning in cell-cell and cellextracellular matrix (cell-ECM) adhesion, cell motility, proliferation, and tumor immunity. Gene expression profiling by DNA microarray revealed differential expression (of $>2$-fold) of about 1000 genes. The weak correlation observed between protein and mRNA profiles highlights the important complementarities of DNA microarray and proteomics approaches. These comparative data enabled us to map the disease-perturbed cell-cell and cell-ECM adhesion and Rho GTPase-mediated cytoskeletal pathways. Further validation of a subset of genes suggests the potential use of vimentin and galectin 1 as markers for metastasis. We demonstrate that combining proteomic and genomic approaches not only provides a rapid, robust, and sensitive platform to elucidate the molecular mechanisms underlying gastric cancer metastasis but also may identify candidate diagnostic markers and therapeutic targets.
\end{abstract}

Keywords: $\quad$ cICAT • 2D-LC-MS/MS • Mass Spectrometry • Gastric Cancer • Metastasis

\section{Introduction}

Gastric cancer is one of the most common malignancies and a leading cause of cancer-related deaths worldwide. ${ }^{1}$ Although the incidence of and mortality due to gastric cancer have

* To whom correspondence should be addressed. For Y.-J. C.: e-mail, yjchen@chem.sinica.edu.tw; phone, 886-2-2789-8660, fax, 886-2-2783-1237.

${ }^{\dagger}$ Institute of Chemistry and Genomic Research Center, Academia Sinica.

${ }^{\ddagger}$ National Taiwan University.

\$ National Yang-Ming University.

" National Taiwan Ocean University.

${ }^{\perp}$ Institute of Biomedical Sciences, Academia Sinica.

○ National Defense University.

\# National Chung Hsing University. declined over the past 40 years $^{2}$ and advances in early diagnosis provide excellent long-term survival probabilities for early stage patients, the overall 5-year survival rate remains less than $10 \%{ }^{3}$ The poor prognosis is mainly due to the fact that most patients are diagnosed at an advanced stage. Advanced gastric cancer is accompanied by metastasis to the peritoneum, lymph nodes, or other organs. ${ }^{4}$ However, the molecular mechanisms associated with tumor metastasis are still not fully understood. Tumor metastasis is mediated by highly complex, multistep processes beginning with loss of adhesion of the tumor cells to neighboring cells. ${ }^{5}$ These cells dissolve the extracellular matrix (ECM), and/or travel via the circulation to invade and proliferate at 
distant new sites. The detailed molecular and cellular mechanisms of carcinogenesis, cancer invasion, and metastasis remain to be elucidated.

Mapping the cellular networks perturbed during cancer progression by systematic, high-throughput, genome-wide expression analysis at both the mRNA and protein levels will facilitate our understanding of these processes. ${ }^{6,7}$ However, it is now generally recognized that mRNA microarray results should be considered preliminary data that require independent follow-up validation. In contrast, a change in protein patterns in a given disease state should reflect the pathologic processes taking place within the cells. The highly complex feedback between the transcriptome and proteome should ideally describe most biochemical processes within and between cells. Thus, a combination of genomic and proteomic analyses is essential. ${ }^{7}$

While substantial efforts have been made to conduct comparative proteomic characterizations of gastric adenocarcinoma ${ }^{8-13}$ most of the efforts to date have used two-dimensional gel electrophoresis (2-DE) and mass spectrometry (MS). Other than the comprehensive account of the proteins thought to have altered expression in gastric cancer, only a limited number of tumor-associated proteins have been observed to be differentially expressed, because of the inherent limitations of detecting extreme $\mathrm{pH} /$ mass and low-abundance proteins. Compared to a 2-DE strategy, protein shotgun sequencing based on two-dimensional liquid chromatography tandem mass spectrometry (2D-LC-MS/MS) analysis represents a large-scale, robust, and sensitive technology for protein profiling. ${ }^{14-16}$ An alternative proteome profiling approach, isotope-coded affinity tagging (ICAT), combined with the shotgun profiling method, allows quantitative analysis of proteomic changes in response to cellular perturbations with a wide dynamic range and good quantitative accuracy. ${ }^{16,17}$ More recently, a second-generation cleavable ICAT (cICAT) reagent was developed to eliminate chromatographic isotope effects caused by hydrogen and deuterium. ${ }^{18}$ Furthermore, the combination of cICAT technology and 2D-LC-MS/MS has been shown to be capable of detecting a large number of proteins, allowing more comprehensive coverage of the proteome. ${ }^{19}$

To study gastric cancer metastasis systematically, we performed a quantitative comparison between the nonmetastatic SC-M1 and metastatic TMC-1 gastric cancer cell lines. Although direct analysis of clinical samples is most appropriate in principle, to provide phenotype information, the heterogeneous genetics of clinical samples requires analysis of a large number of patients to guarantee statistical significance. Therefore, wellcharacterized cell lines may prove more informative because of advantages in reproducibility, availability of large numbers, and genetic homogeneity. SC-M1 is a well-established human gastric carcinoma cell line, originally cultured from a poorly differentiated adenocarcinoma that invaded through the stomach wall but showed no metastasis to lymph nodes or adjacent organs. ${ }^{20}$ Another gastric cancer cell line, TMC-1, was derived from the lymph node of an individual with a moderately differentiated adenocarcinoma of the stomach and exhibited highly tumorigenic features. ${ }^{21}$ The comparative mRNA and protein profiling of these two cell lines may allow us to study the cellular processes associated with the development of gastric cancer metastasis, facilitating further investigation by clinical specimens.

As shown in Figure 1, we first performed qualitative profiling to define the protein constituents of SC-M1 and TMC-1 cells

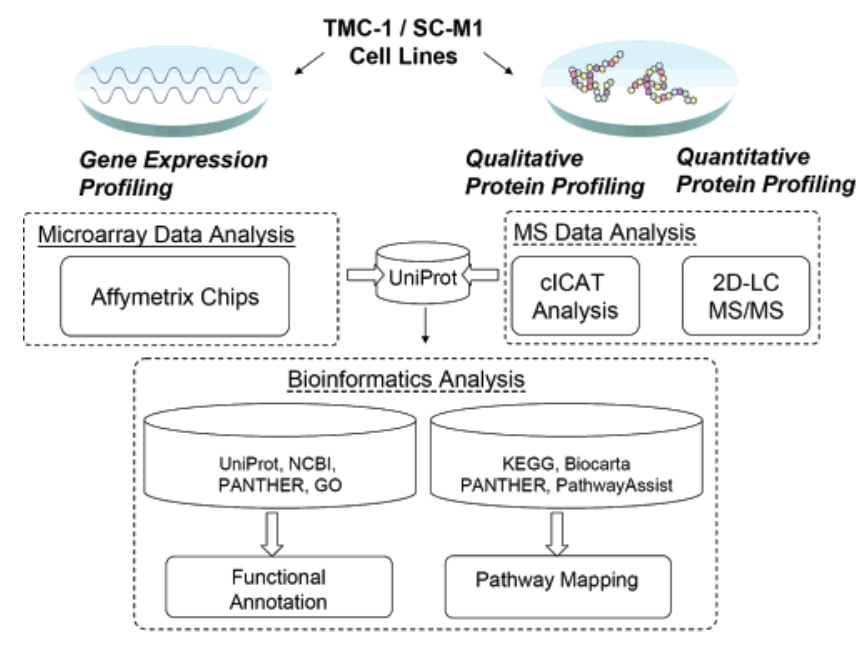

Figure 1. Schematic representation of the experimental design and bioinformatics analysis for comprehensive genomic and proteomic profiling of SC-M1 and TMC-1 cells.

by $2 \mathrm{D}-\mathrm{LC}-\mathrm{-S} / \mathrm{MS}$. We further compared protein and mRNA expression profiles between SC-M1 and TMC-1 cells using cICAT technology and Affymetrix DNA microarrays. To our knowledge, our results offer a more comprehensive proteomic analysis of gastric cancer cells than previously reported. By the complementary analysis of differentially expressed proteins and mRNAs in the two cell lines, we aimed to address the following two issues: (1) the elucidation of the molecular mechanism of metastasis of gastric cancer and (2) the identification of potential markers and/or expression signatures for prognosis and drug discovery for future therapeutic intervention. While the current study focuses on gastric cancer, we expect that the potential markers and pathways uncovered here may be relevant to the metastatic phenotypes of cancer in general.

\section{Materials and Methods}

Chemicals. RPMI 1640 medium was purchased from HyClone (Logan, UT). Fetal bovine serum (FBS) was purchased from Gibco BRL (Grand Island, NY). Trypsin was purchased from Promega (Madison, WI). Tris (2-carboxyethyl) phosphine (TCEP) was obtained from Fisher Scientific Corp. (Pittsburgh, PA). The BCA protein assay kit was obtained from Pierce (Rockford, IL). Tris, SDS, Triton X-100, trifluoroacetic acid (TFA), formic acid, and acetonitrile were purchased from Sigma-Aldrich (St. Louis, MO). Iodoacetamide was purchased from Amersham Biosciences (England, U.K.)

Cell Culture and Lysis. The human gastric cancer cell lines SC-M1 and TMC-1 were grown in RPMI 1640 medium supplemented with $25 \mathrm{mM}$ HEPES, $26 \mathrm{mM} \mathrm{NaHCO}_{3}, 2 \mathrm{mM}$ glutamine, 100 units/mL sodium penicillin $\mathrm{G}, 100 \mu \mathrm{g} / \mathrm{mL}$ streptomycin sulfate, $0.25 \mu \mathrm{g}$ amphotericin B (fungizone), and $10 \%$ fetal bovine serum (FBS, Invitrogen Corporation, Carlsbad, CA) at $37{ }^{\circ} \mathrm{C}$ with $5 \% \mathrm{CO}_{2}$. The cells were harvested, washed, divided into aliquots containing about $\sim 2 \times 10^{7}$ cells each, and suspended in lysis buffer (50 mM Tris at pH $8.0,0.1 \%(\mathrm{w} / \mathrm{v})$ SDS, $0.02 \%$ (w/v) Triton X-100). The suspensions were lysed by placement into a sonic water bath for $30 \mathrm{~min}$ followed by exposure to three liquid nitrogen freeze-thaw cycles. Total protein concentration of each cell extract was determined using the BCA assay.

Qualitative Protein Profiling by 2D-LC-MS/MS. About 1 mg of total protein from each cell extract was reduced by TECP, 
alkylated by IAA, and digested by trypsin. Strong cation exchange (SCX) chromatography was used to fractionate the tryptic peptides. The tryptic peptides were fractionated using a $2.1 \times 200 \mathrm{~mm}$ polysulfoethyl A SCX column (Poly LC, Columbia, MD). Peptides were eluted with a gradient of $0-25 \%$ buffer B $\left(5 \mathrm{mM} \mathrm{K}_{2} \mathrm{HPO}_{4}, 25 \%\right.$ acetonitrile, and $350 \mathrm{mM}, \mathrm{KCl}$, $\mathrm{pH} 3.0$ ) over $30 \mathrm{~min}$, followed by a gradient of $25-100 \%$ buffer B over $20 \mathrm{~min}$. The SCX fractions (retention time: $20-60 \mathrm{~min}$ ) were analyzed in duplicate by microcapillary-liquid chromatography MS/MS ( $\mu \mathrm{LC}-\mathrm{MS} / \mathrm{MS}$ ).

Quantitative Protein Profiling by cICAT and 2D-LC-MS/ MS. About $400 \mu \mathrm{g}$ of total protein extracted from TMC-1 and SC-M1 cells was labeled with heavy and light isotope-label cICAT, respectively. The cICAT labeling reaction was performed following the manufacturer's protocol (Applied Biosystems, Framingham, MA). The labeled samples were combined and subjected to the trypsin digestion. The digested samples were further fractionated by SCX chromatography, and the cICATlabeled peptides were isolated by avidin-affinity chromatography for subsequent $\mu \mathrm{LC}-\mathrm{MS} / \mathrm{MS}$ analysis.

$\boldsymbol{\mu} \mathbf{L C}-\mathbf{M S} / \mathbf{M S}$ Analysis. Samples were loaded using an autosampler and injected into a $1.5 \mathrm{~cm} \times 100 \mu \mathrm{m}$ trapping column and $12 \mathrm{~cm} \times 75 \mu \mathrm{m}$ separation column packed in-house (Magic $\mathrm{C}_{18}$; Michrom BioResources, Auburn, CA) and sequentially analyzed by $\mu \mathrm{LC}-\mathrm{MS} / \mathrm{MS}$. Peptides were eluted with a linear gradient of $5-40 \%$ buffer B over 120 min at $\sim 200$ $\mathrm{nL} / \mathrm{min}$ (buffer $\mathrm{A}, 0.1 \%$ formic acid in $\mathrm{H}_{2} \mathrm{O}$; buffer $\mathrm{B}, 0.1 \%$ formic acid in acetonitrile). An HP 1100 solvent delivery system (Hewlett-Packard, Palo Alto, CA) was used with precolumn flow splitting. A quadrupole/time-of-flight mass spectrometer (QSTAR Pulsar $i$, Applied Biosystems, Foster City, CA) with an in-house built microspray device was used for all analyses. Peptide fragmentation by collision-induced dissociation was performed in an automated fashion using the information-dependent option.

Data Processing. Uninterpreted MS/MS spectra were searched against the Celera Discovery System (CDS, Celera Genomics, CA) sequence classification protein database (version date 08/ 13/2003) using the TurboSEQUEST (v.27-rev.12, ThermoFinnigan, San Jose, CA) and validated by the PeptideProphet. ${ }^{22}$ The peptides that displayed a PeptideProphet score $>0.7$ were analyzed by ProteinProphet ${ }^{23}$ to calculate the false-positive score of each identified protein. The proteins or related protein groups were identified with a probability score cutoff of $p>$ 0.95 . For the qualitative analysis, this value corresponded to a false-positive error rate of 0.005 and 0.004 for SC-M1 and TMC1 , respectively. For the cICAT analysis, this value corresponded to a false-positive error rate of 0.004 . The light-to-heavy mass $(L / H)$ ratio resulting from differential expression was calculated and normalized by ASAPRatio. ${ }^{24}$ All of the cICAT-labeled peptides quantified by ASAPRatio were also verified manually.

Microarray Analysis. Total cellular RNA was extracted from a minimum of $5 \times 10^{6}$ cells using the TRIZOL reagent (Invitrogen Corp.). RNA was additionally purified by phenol/ chloroform/isoamyl alcohol (25:24:1) extraction, and RNA quality was checked using an Agilent 2100 Bioanalyzer (Agilent Technologies, Foster City, CA). RNA processing and hybridization to the Affymetrix HG-U133A GeneChip oligonucleotide microarray (Santa Clara, CA) were performed according to the manufacturer's protocol. Initial data analysis was performed using Microarray Suite v 5.0 software (Affymetrix, Santa Clara, CA), setting the scaling of all probe sets to a constant value of 500 for each GeneChip. Additional data analysis was performed using GeneSpring v 5.1 (Silicon Genetics Inc., Redwood City, CA). Genes with differential expression levels of 2-fold or more between SC-M1 and TMC-1 cells were selected for further analysis.

Functional Classification and Pathway Annotations. Functional classification of the identified proteins was mainly based on the CDS sequence classification protein database (version date $08 / 13 / 2003$ ). The proteins without functional annotation in the CDS database were searched against the UniProt (http:// www.pir.uniprot.org/), NCBI (http://www.ncbi.nlm.nih.gov/), PANTHER (http://www.pantherdb.org/), and Gene Ontology (GO) (http://www.geneontology.org/) consortium databases to obtain their biological functions.

For analysis of the perturbed pathway of each differentially expressed gene and protein, we retrieved 314 BioCarta (http:// www.biocarta.com) and 155 Kyoto Encyclopedia of Genes and Genome (KEGG, http://www.genome.ad.jp/kegg/pathway. html) pathways. The Unigene accession numbers were used as identifiers to compare with the proteomic and microarray data. The proteomic data is also analyzed by the pathway analysis tool in NCI's Cancer Genome Anatomy Project (CGAP, http://cgap.nci.nih.gov/Pathways).

Immunocytostaining. For immunocytostaining studies, cells grown on coverslips were fixed in $4 \%$ paraformaldehyde for $30 \mathrm{~min}$ at room temperature, washed twice with TBS (50 mM Tris- $\mathrm{HCl}$ and $150 \mathrm{mM} \mathrm{NaCl}, \mathrm{pH}$ 7.4), and blocked in TBBS (50 mM Tri-HCl, pH 7.4, $150 \mathrm{mM} \mathrm{NaCl,} \mathrm{0.1 \%} \mathrm{Triton} \mathrm{X-100,} \mathrm{and}$ $3 \%$ bovine serum albumin) for $1 \mathrm{~h}$ at $37^{\circ} \mathrm{C}$. $\beta$-Catenin was detected using anti- $\beta$-catenin rabbit polyclonal antibody purchased from Sigma (St. Louis, MO) in conjunction with Alexa Fluor 488-conjugated anti-rabbit IgG secondary antibody purchased from Invitrogen. Cells were also counter-stained with 4,6-diamidino-2-phenylindole-dihydrochloride (DAPI, Vector Laboratories, Inc., Burlingame, CA). Cells were analyzed by a laser scanning confocal system (Radiance-2100; Bio-Rad, Hercules, CA).

Flow Cytometry. Cells were seeded at $1 \times 10^{5}$ cells/well in 6 -well microplates and incubated overnight in DMEM containing $10 \%$ fetal calf serum. Cells were trypsinized; stained with a monoclonal antibody (mAb) against integrin $\alpha 6$ (CD49f, Serotec, Oxford, U.K.), integrin $\beta 4$ (Serotec), or an isotype IgG; washed with cold PBS three times; labeled with Alexa Fluor 488-conjugated anti-mouse IgG antibody; and subjected to flow cytometry analysis using a FACSCalibur instrument (BD Biosciences). The specific fluorescence index was calculated as the ratio of the mean fluorescence values obtained with the specific $\mathrm{Ab}$ and the isotype control $\mathrm{Ab}$.

Alamar Blue Assay for Cell Proliferation and Viability. Cells in a 96-well plate were incubated for $4 \mathrm{~h}$ with Alamar Blue dye (Biosource) to measure cellular proliferation and viability. Dye uptake was measured fluorometrically using an ELISA Reader (Molecular Devices Corporation, CA) at an excitation and emission wavelength of 570 and $600 \mathrm{~nm}$, respectively.

\section{Results}

Qualitative Protein Profiling of SC-M1 and TMC-1 Cell Lines. As shown in Figure 1, qualitative profiling was performed by 2D-LC-MS/MS to define the protein constituents in SC$\mathrm{M} 1$ and TMC-1 cells prior to quantitative profiling experiments and to facilitate detection of low-abundance proteins and/or cysteine-deficient proteins that cannot be observed by cICAT analysis. Using a stringent identification criterion (protein identification false-positive rate of $<0.005$ ), 926 and 909 protein 
A

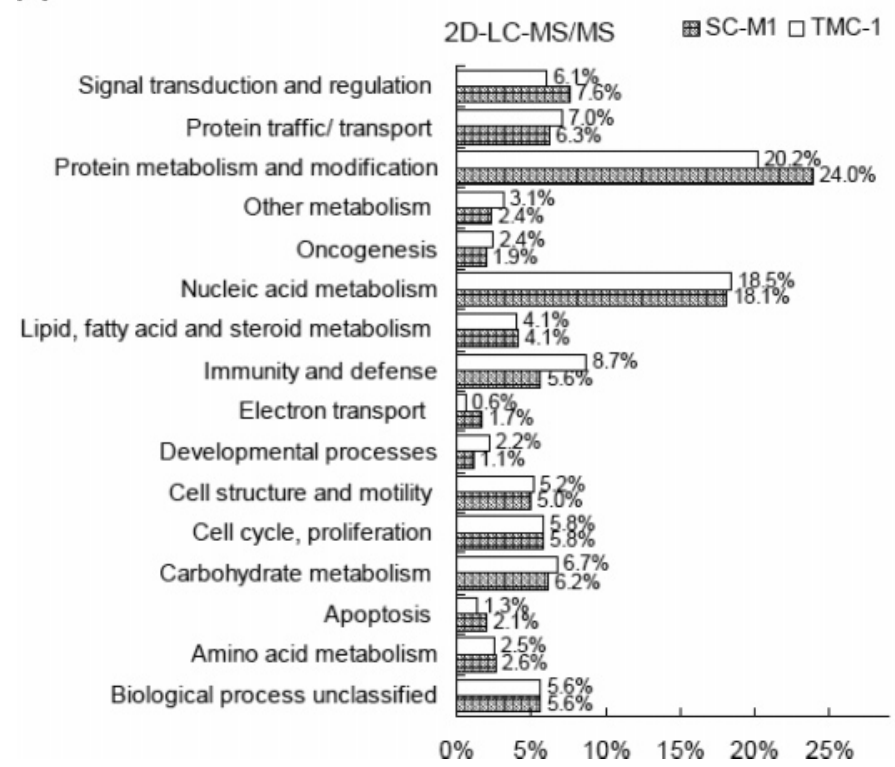

B

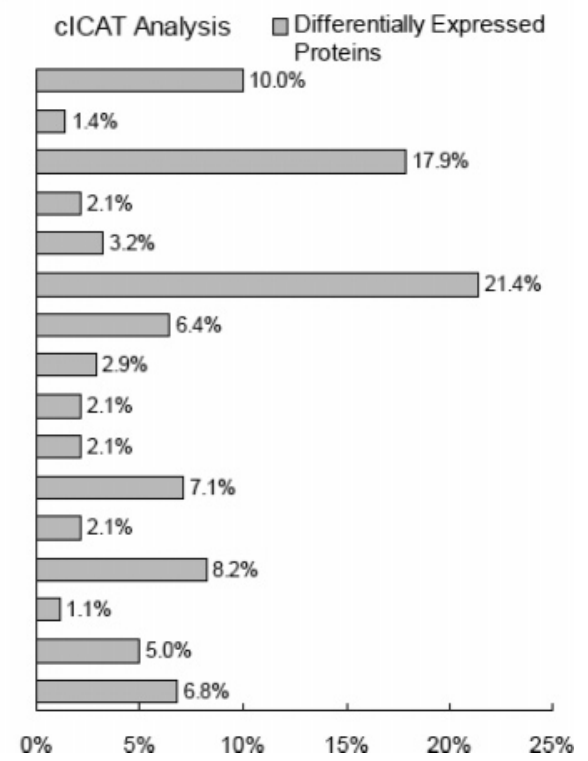

Figure 2. Functional classification of (A) SC-M1 and TMC-1 proteins from 2D-LC-MS/MS. (B) Differentially expressed proteins between SC-M1 and TMC-1 cells from cICAT analysis. The numbers indicate the percentage of proteins that were differentially expressed between the two cell lines for each functional classification.

entries from SC-M1 and TMC-1 cells were identified, respectively (Supplemental Tables 1 and 2, Supporting Information). For those proteins identified in the qualitative profiling, 41 and 48 proteins were cysteine-deficient in SC-M1 and TMC-1, respectively.

To obtain an overview of the similarities and differences in protein expression between the cell lines, functional annotation was performed to compare the protein constituents. Figure 2A compares the functional category distribution of SC-M1 and TMC-1 proteins according to biological processes (also see Supplemental Tables 1 and 2, Supporting Information). As expected, the identified proteins in the two cell lines possess similar functional category patterns. Most proteins fit into two main categories: protein metabolism and modification, and nucleic acid metabolism. A majority of the other proteins participate in signal transduction and regulation, cell structure and mobility, cell cycle and proliferation, immunity and defense, and protein traffic and transport. Several proteins were classified to be oncogenesis-associated, including 14-3-3 protein $\sigma$, 14-3-3 protein $\zeta, 14-3-3$ protein $\tau$, annexin I, galectin 1, myc box-dependent interacting protein 1 , and T-plastin; these proteins have also been previously found to be differentially expressed in cancer cells. ${ }^{8,25-27}$

Differential Proteomic and Transcriptomic Analyses of SCM1 and TMC-1 Cell Lines. In cICAT analysis, 1727 cICATlabeled peptides originated from 615 proteins were identified (with a protein identification false-positive rate of $<0.005$ ), of which 559 could be quantified. From the global mean and standard deviation in this study, we have chosen to highlight proteins that have abundance ratios greater than 1.3-fold. Using this criterion, we identified 240 proteins that displayed more than a 1.3-fold expression difference; 100 proteins were upregulated and 140 proteins were down-regulated in the TMC-1 cells as compared with the SC-M1 cells. Table 1 lists the summary of these differentially expressed proteins. Figure $2 \mathrm{~B}$ displays the functional category distribution of differentially expressed proteins. Other than metabolism, prominent among the differential expression findings were proteins involved in signal transduction and regulation, including down-regulation of the Rho-related GTP-binding protein RhoG, transforming protein RhoA, and transforming protein RhoC in TMC-1 cells. Other significant changes involved proteins related to cell structure and motility. These functional categories showed higher percentage of distributions in comparison with qualitative profiling of SC-M1 and TMC-1 (Figure 2A). Figure 3 shows a differentially expressed protein, $\beta$-catenin, quantified by cICAT analysis. The light and heavy cICAT-labeled peptide revealed a 5.9-fold overexpression in TMC-1 cells compared with SC-M1 cells (Figure 3B,C). This observation was confirmed with Western blotting, as shown in Figure 3D.

To examine whether the differential protein expression may be attributable to transcriptional regulation or another regulatory mechanism, mRNA expression profiling was performed on SC-M1 and TMC-1 cells using an Affymetrix HG-U133A GeneChip oligonucleotide microarray. The pathways containing differentially expressed genes are listed in Table 2. Among these pathways, three are involved in cancer transformation and proliferation: inhibition of matrix metalloproteinases, multidrug resistance (MDR) factors, and thrombospondin-1 (TSP1)-induced apoptosis. Matrix metalloproteinases are a class of proteases secreted by tumor cells that degrade the proteins of the ECM for subsequent tumor cell invasion and metastasis. There are many MDR genes among the mRNAs differentially expressed in SC-M1 and TMC-1 cells, including members of the MDR/TAP subfamily, cytochrome P450 51A1, and glutathione-S-transferase. ${ }^{28}$ TSP-1 inhibits angiogenesis and slows tumor growth, apparently by inducing apoptosis of microvascular endothelial cells that line blood vessels. ${ }^{29}$ Factors that inhibit angiogenesis might act as cancer therapeutic agents by blocking vessel formation in tumors and starving cancer cells. These pathways may be relevant to the differences between SC-M1 and TMC-1 cells.

Transcriptional versus Translational Regulation of Protein Expression. Changes in protein expression levels between the two cancer cell types were further examined for potential correlations with the changes in mRNA levels. Cross-referencing 
Table 1. Summary of Differentially Expressed Proteins and Their Functional Classifications

\begin{tabular}{|c|c|c|c|c|c|c|c|}
\hline & UniProt & protein name & $\begin{array}{l}\text { peptide } \\
\text { number }\end{array}$ & $\operatorname{prob}^{a}$ & $\operatorname{cICAT}^{b}$ & Affymetrix $^{c}$ & molecular function \\
\hline \multicolumn{8}{|c|}{ Amino Acid Metabolism } \\
\hline 1 & P31327 & Carbamoyl-phosphate synthase & 2 & 1.00 & $0.16 \pm 0.03$ & $\mathrm{NA}^{d}$ & Synthase and synthetase \\
\hline 2 & $\mathrm{O} 43175$ & D-3-phosphoglycerate dehydrogenase & 6 & 1.00 & $0.31 \pm 0.07$ & 0.52 & Oxidoreductase \\
\hline 3 & P26639 & Threonyl-tRNA synthetase, cytoplasmic & 2 & 1.00 & $0.35 \pm 0.04$ & 0.92 & Synthase \\
\hline 4 & P11586 & C-1-tetrahydrofolate synthase & 2 & 1.00 & $0.43 \pm 0.17$ & 0.58 & Synthase and synthetase \\
\hline 5 & P35520 & Cystathionine beta-synthase & 2 & 0.99 & $0.44 \pm 0.07$ & 0.45 & Synthase \\
\hline 6 & Q9BWD1 & Acetyl CoA transferase-like protein & 2 & 1.00 & $0.52 \pm 0.03$ & 1.00 & Molecular function unclassified \\
\hline 7 & P54886 & Delta 1-pyrroline-5-carboxylate synthetase & 2 & 1.00 & $0.55 \pm 0.08$ & 0.58 & Kinase \\
\hline 8 & P30046 & D-dopachrome tautomerase & 2 & 1.00 & $0.56 \pm 0.05$ & 0.59 & Isomerase \\
\hline 9 & 095671 & $\mathrm{~N}$-acetylserotonin $\mathrm{O}$-methyltransferase-like protein & 1 & 0.96 & $1.39 \pm 0.12$ & 1.25 & Transferase \\
\hline 10 & Q9BSE5 & Agmatinase, mitochondrial precursor & 2 & 1.00 & $1.54 \pm 0.15$ & $\mathrm{NA}^{d}$ & Hydrolase \\
\hline 11 & Q9UJ54 & DTDP-4-keto-6-deoxy-D-glucose 4-reductase & 2 & 1.00 & $1.63 \pm 0.14$ & 1.27 & Oxidoreductase \\
\hline 12 & Q9Y617 & Phosphoserine aminotransferase & 1 & 0.98 & $1.78 \pm 0.41$ & 1.54 & Transferase \\
\hline 13 & P00505 & Aspartate aminotransferase, mitochondrial precursor & 1 & 0.95 & $1.84 \pm 0.13$ & 0.37 & Transferase \\
\hline 14 & P27708 & $\mathrm{CAD}$ protein & 3 & 1.00 & $2.18 \pm 0.53$ & 1.44 & Synthase and synthetase \\
\hline \multicolumn{8}{|c|}{ Apoptosis } \\
\hline 1 & O43293 & Prostate apoptosis response protein par- 4 & 1 & 1.00 & $0.31 \pm 0.03$ & 1.27 & kinase \\
\hline 2 & Q6FI81 & PRO0915 & 1 & 0.99 & $0.59 \pm 0.20$ & $\mathrm{NA}^{d}$ & Select regulatory molecule \\
\hline 3 & O95831 & Programmed cell death protein 8 , mitochondrial precursor & 1 & 1.00 & $2.77 \pm 0.80$ & 1.70 & Oxidoreductase \\
\hline \multicolumn{8}{|c|}{ Carbohydrate Metabolism } \\
\hline 1 & P15121 & Aldose reductase & 2 & 1.00 & $0.08 \pm 0.04$ & NAd & Reductase \\
\hline 2 & P47895 & Aldehyde dehydrogenase 6 & 1 & 0.99 & $0.13 \pm 0.03$ & $\mathrm{NA}^{d}$ & Hydrolase \\
\hline 3 & P19367 & Hexokinase, type I & 3 & 1.00 & $0.31 \pm 0.08$ & 0.29 & Kinase \\
\hline 4 & P29401 & Transketolase & 1 & 0.99 & $0.31 \pm 0.03$ & 0.45 & Transferase \\
\hline 5 & P36871 & Phosphoglucomutase & 1 & 0.99 & $0.37 \pm 0.34$ & 0.23 & Isomerase \\
\hline 6 & Q01813 & 6-phosphofructokinase, type C & 3 & 1.00 & $0.43 \pm 0.09$ & 0.31 & Kinase \\
\hline 7 & P18669 & Phosphoglycerate mutase 1 & 2 & 1.00 & $0.44 \pm 0.04$ & 0.47 & Isomerase \\
\hline 8 & P14786 & Pyruvate kinase, M2 isozyme & 4 & 1.00 & $0.51 \pm 0.09$ & 0.52 & kinase \\
\hline 9 & Q92621 & Hypothetical protein KIAA0225 & 3 & 1.00 & $0.51 \pm 0.13$ & 0.50 & Kinase \\
\hline 10 & P00338 & L-lactate dehydrogenase A chain & 3 & 1.00 & $0.65 \pm 0.03$ & 0.83 & Oxidoreductase \\
\hline 11 & P16152 & Carbonyl reductase [NADPH] 1 & 2 & 1.00 & $0.65 \pm 0.03$ & $\mathrm{NA}^{d}$ & Reductase \\
\hline 12 & P53396 & ATP-citrate synthase & 3 & 1.00 & $0.69 \pm 0.04$ & 0.80 & Lyase \\
\hline 13 & P48735 & Isocitrate dehydrogenase [NADP], mitochondrial precursor & 2 & 1.00 & $1.33 \pm 0.11$ & 0.51 & Oxidoreductase \\
\hline 14 & P14550 & Alcohol dehydrogenase $[\mathrm{NADP}+]$ & 1 & 1.00 & $1.36 \pm 0.24$ & 0.86 & Oxidoreductase \\
\hline 15 & P42330 & Aldo-keto reductase family 1 member C3 & 2 & 1.00 & $1.56 \pm 0.10$ & 2.68 & Oxidoreductase \\
\hline 16 & P52789 & Hexokinase, type II & 3 & 1.00 & $1.66 \pm 0.16$ & 1.15 & Kinase \\
\hline 17 & Q02218 & 2-oxoglutarate dehydrogenase E1 component, mitochondrial precursor & 2 & 1.00 & $1.67 \pm 0.39$ & 2.24 & Oxidoreductase \\
\hline 18 & P37837 & Transaldolase & 2 & 1.00 & $1.78 \pm 0.19$ & 1.29 & Transferase \\
\hline 19 & Q00796 & Sorbitol dehydrogenase & 1 & 1.00 & $1.90 \pm 0.21$ & 3.04 & Oxidoreductase \\
\hline 20 & P10768 & Esterase D & 2 & 1.00 & $2.21 \pm 1.09$ & 1.56 & Hydrolase \\
\hline 21 & P40925 & Malate dehydrogenase, cytoplasmic & 2 & 1.00 & $2.24 \pm 0.12$ & 1.03 & Oxidoreductase \\
\hline 22 & P40926 & Malate dehydrogenase, mitochondrial precursor & 11 & 1.00 & $4.49 \pm 0.60$ & 2.60 & Oxidoreductase \\
\hline 23 & O60547 & GDP-mannose 4,6 dehydratase & 1 & 1.00 & $6.15 \pm 1.02$ & 3.09 & Lyase \\
\hline \multicolumn{8}{|c|}{ Cell Cycle, Proliferation } \\
\hline 1 & O75369 & Beta-filamin & 13 & 1.00 & $0.20 \pm 0.09$ & 0.08 & Cytoskeletal protein \\
\hline 2 & P58107 & Epiplakin & 1 & 1.00 & $0.21 \pm 0.04$ & $\mathrm{NA}^{d}$ & Cytoskeletal protein \\
\hline 3 & Q15691 & Microtubule-associated protein RP/EB family member 1 & 1 & 0.98 & $0.48 \pm 0.09$ & 0.98 & Signaling molecule \\
\hline 4 & Q14980 & NuMA protein & 2 & 1.00 & $0.68 \pm 0.18$ & 0.45 & Cytoskeletal protein \\
\hline 5 & Q14683 & Structural maintenance of chromosome 1-like 1 protein & 1 & 1.00 & $0.68 \pm 0.33$ & 0.77 & Nucleic acid binding \\
\hline 6 & Q9Y2Z0 & Suppressor of G2 allele of SKP1 homolog & 1 & 1.00 & $2.29 \pm 0.20$ & $\mathrm{NA}^{d}$ & Molecular function unclassified \\
\hline \multicolumn{8}{|c|}{ Cell Structure and Motility } \\
\hline 1 & Q04695 & Cytoskeletal 17 & 2 & 1.00 & $0.03 \pm 0.03$ & $\mathrm{NA}^{d}$ & Membrane \\
\hline 2 & Q96AC1 & Hypothetical protein & 1 & 1.00 & $0.04 \pm 0.00$ & 0.04 & protein binding \\
\hline 3 & Q9NZM1 & Myoferlin & 2 & 1.00 & $0.17 \pm 0.08$ & 0.40 & Membrane traffic protein \\
\hline 4 & Q9P2E9 & Ribosome-binding protein 1 & 4 & 1.00 & $0.38 \pm 0.26$ & $\mathrm{NA}^{d}$ & Receptor \\
\hline 5 & P35579 & Myosin heavy chain, nonmuscle type A & 7 & 1.00 & $0.47 \pm 0.05$ & 0.38 & Cytoskeletal protein \\
\hline 6 & P24572 & Myosin light chain alkali, smooth-muscle isoform & 1 & 1.00 & $0.48 \pm 0.03$ & 0.63 & Select regulatory molecule \\
\hline 7 & P52907 & F-actin capping protein alpha- 1 subunit & 2 & 1.00 & $0.54 \pm 0.05$ & 0.86 & Cytoskeletal protein \\
\hline 8 & P07737 & Profilin I & 3 & 1.00 & $0.63 \pm 0.09$ & 0.10 & Cytoskeletal protein \\
\hline 9 & P47756 & F-actin capping protein beta subunit & 2 & 1.00 & $0.63 \pm 0.09$ & 0.86 & Cytoskeletal protein \\
\hline 10 & Q8NAG3 & Hypothetical protein FLJ35393 & 1 & 1.00 & $0.63 \pm 0.29$ & $\mathrm{NA}^{d}$ & Cytoskeletal protein \\
\hline 11 & Q9Y3F5 & A6 related protein & 1 & 1.00 & $0.68 \pm 0.13$ & $\mathrm{NA}^{d}$ & Cytoskeletal protein \\
\hline 12 & O75083 & WD-repeat protein 1 & 2 & 1.00 & $0.69 \pm 0.12$ & 0.58 & Cytoskeletal protein \\
\hline 13 & O43707 & Alpha-actinin 4 & 1 & 1.00 & $1.37 \pm 0.07$ & 1.59 & Cytoskeletal protein \\
\hline 14 & P21333 & Filamin A & 11 & 1.00 & $1.44 \pm 0.07$ & 1.87 & Cell adhesion molecule \\
\hline 15 & P15924 & Desmoplakin & 3 & 1.00 & $1.57 \pm 0.12$ & 2.07 & Cytoskeletal protein \\
\hline 16 & P02545 & Lamin A/C & 1 & 0.99 & $2.11 \pm 0.72$ & 0.90 & Cytoskeletal protein \\
\hline 17 & P27816 & Microtubule-associated protein 4 & 1 & 0.95 & $2.29 \pm 0.37$ & 0.66 & Cytoskeletal protein \\
\hline
\end{tabular}


Table I (Continued)

\begin{tabular}{|c|c|c|c|c|c|c|c|}
\hline & UniProt & protein name & $\begin{array}{l}\text { peptide } \\
\text { number }\end{array}$ & prob. ${ }^{a}$ & $\operatorname{cICAT}^{b}$ & Affymetrix ${ }^{c}$ & molecular function \\
\hline \multicolumn{8}{|c|}{ Cell Structure and Motility } \\
\hline 18 & Q9NQW6 & Actin binding protein anillin & 1 & 1.00 & $2.58 \pm 0.45$ & $\mathrm{NA}^{d}$ & Cytoskeletal protein \\
\hline 19 & P13647 & Cytoskeletal 5 & 1 & 1.00 & $3.43 \pm 1.10$ & $\mathrm{NA}^{d}$ & Cytoskeletal protein \\
\hline 20 & P08670 & Vimentin & 1 & 0.99 & $25.67 \pm 5.81$ & $\mathrm{NA}^{d}$ & Cytoskeletal protein \\
\hline \multicolumn{8}{|c|}{ Developmental Processes } \\
\hline 1 & P21291 & Cysteine-rich protein 1 & 1 & 1.00 & $0.3 \pm 0.04$ & 0.59 & Ion binding \\
\hline 2 & Q14192 & Skeletal muscle LIM-protein 3 & 1 & 0.93 & $0.54 \pm 0.09$ & 0.92 & Transcription factor \\
\hline 3 & Q09666 & Neuroblast differentiation associated protein AHNAK & 2 & 1.00 & $0.67 \pm 0.13$ & 0.44 & Molecular function unclassified \\
\hline 4 & Q15668 & Epididymal secretory protein E1 precursor & 1 & 1.00 & $0.63 \pm 0.04$ & 0.23 & Signaling molecule \\
\hline 5 & Q9HDC9 & Adipocyte plasma membrane-associated protein & 1 & 1.00 & $1.36 \pm 0.42$ & 0.82 & Molecular function unclassified \\
\hline 6 & Q16658 & Fascin & 4 & 1.00 & $2.98 \pm 0.39$ & 1.07 & Cytoskeletal protein \\
\hline \multicolumn{8}{|c|}{ Electron Transport } \\
\hline 1 & P23634 & Plasma membrane calcium-transporting ATPase 4 & 1 & 0.99 & $0.00 \pm 0.00$ & 0.06 & Hydrolase \\
\hline 2 & P08574 & Cytochrome $c$, heme protein, mitochondrial precursor & 1 & 1.00 & $0.68 \pm 0.04$ & 0.82 & Oxidoreductase \\
\hline 3 & Q9NQ11 & Hypothetical protein & 1 & 0.99 & $1.41 \pm 0.11$ & $\mathrm{NA}^{d}$ & Hydrolase \\
\hline 4 & P22695 & Ubiquinol-cytochrome $\mathrm{C}$ reductase complex core protein 2 & 2 & 1.00 & $1.56 \pm 0.24$ & 0.72 & Reductase \\
\hline 5 & P51970 & NADH-ubiquinone oxidoreductase 19 kDa subunit & 2 & 1.00 & $1.90 \pm 0.36$ & 1.34 & Oxidoreductase \\
\hline 6 & 096000 & NADH-ubiquinone oxidoreductase PDSW subunit & 1 & 0.92 & $2.17 \pm 0.47$ & $\mathrm{NA}^{d}$ & Oxidoreductase \\
\hline \multicolumn{8}{|c|}{ Immunity Defenses } \\
\hline 1 & O14879 & Interferon-induced protein with tetratricopeptide repeats 4 & 3 & 1.00 & $0.08 \pm 0.05$ & $\mathrm{NA}^{d}$ & Defense/immunity protein \\
\hline 2 & P09914 & Interferon-induced protein with tetratricopeptide repeats 1 & 2 & 1.00 & $0.29 \pm 0.14$ & $\mathrm{NA}^{d}$ & Molecular function unclassified \\
\hline 3 & Q03405 & Urokinase plasminogen activator surface receptor precursor & 2 & 1.00 & $0.42 \pm 0.12$ & 0.16 & Receptor \\
\hline 4 & P30041 & Peroxiredoxin 6 & 2 & 1.00 & $0.55 \pm 0.07$ & 0.67 & Oxidoreductase \\
\hline 5 & P01892 & HLA class I histocompatibility antigen, A-10 alpha chain (Fragment) & 1 & 1.00 & $0.64 \pm 0.16$ & $\mathrm{NA}^{d}$ & Defense/immunity protein \\
\hline 6 & P13987 & CD59 glycoprotein precursor & 1 & 0.98 & $0.67 \pm 0.56$ & 0.39 & Signaling molecule \\
\hline 7 & P30044 & Peroxiredoxin 5, mitochondrial precursor & 1 & 1.00 & $1.84 \pm 0.89$ & $\mathrm{NA}^{d}$ & Oxidoreductase \\
\hline 8 & P09382 & Galectin-1 & 4 & 1.00 & $2.33 \pm 0.72$ & 0.90 & Signaling molecule \\
\hline \multicolumn{8}{|c|}{ Protein Traffic } \\
\hline 1 & Q96C19 & Swiprosin 1 & 2 & 1.00 & $0.34 \pm 0.04$ & 0.28 & Nucleic acid binding \\
\hline 2 & O60664 & Cargo selection protein TIP47 & 1 & 1.00 & $0.55 \pm 0.13$ & 0.40 & Receptor \\
\hline 3 & Q9NR31 & GTP-binding protein SARla & 2 & 1.00 & $0.59 \pm 0.18$ & 0.78 & Select regulatory molecule \\
\hline 4 & O60684 & Importin alpha 7 subunit & 1 & 0.94 & $0.67 \pm 0.09$ & 0.75 & Membrane traffic protein \\
\hline 5 & P05218 & Tubulin beta- 5 chain & 13 & 1.00 & $1.33 \pm 0.22$ & 0.50 & Cytoskeletal protein \\
\hline 6 & Q9BS26 & Thioredoxin domain containing protein 4 precursor & 5 & 1.00 & $1.33 \pm 0.21$ & 0.86 & Isomerase \\
\hline 7 & P55072 & Transitional endoplasmic reticulum ATPase & 8 & 1.00 & $1.35 \pm 0.12$ & 0.95 & Hydrolase \\
\hline 8 & Q14204 & Dynein heavy chain, cytosolic & 3 & 1.00 & $1.44 \pm 0.17$ & 1.03 & Cytoskeletal protein \\
\hline 9 & O00159 & Myosin Ic & 2 & 1.00 & $1.45 \pm 0.64$ & 0.57 & Cytoskeletal protein \\
\hline 10 & P10809 & Heat shock protein 60 & 9 & 1.00 & $1.56 \pm 0.09$ & 1.13 & Chaperon \\
\hline 11 & Q13509 & Tubulin beta- 4 chain & 1 & 0.98 & $1.77 \pm 0.33$ & 0.77 & Cytoskeletal protein \\
\hline 12 & O43765 & Small glutamine-rich tetratricopeptide repeat-containing protein & 2 & 1.00 & $1.88 \pm 0.40$ & $\mathrm{NA}^{d}$ & Chaperon \\
\hline 13 & P30740 & Leukocyte elastase inhibitor & 1 & 0.91 & $2.41 \pm 0.44$ & 3.76 & Select regulatory molecule \\
\hline \multicolumn{8}{|c|}{ Lipid, Fatty Acid, and Steroid Metabolism } \\
\hline 1 & Q99541 & Adipophilin & 1 & 0.99 & $0.01 \pm 0.00$ & N.A. & Molecular function unclassified \\
\hline 2 & Q01581 & Hydroxymethylglutaryl-CoA synthase & 2 & 1.00 & $0.13 \pm 0.03$ & 0.18 & Synthase and synthetase \\
\hline 3 & Q9Y6L9 & Titin & 1 & 0.99 & $0.16 \pm 0.01$ & $\mathrm{NA}^{d}$ & Cytoskeletal protein- \\
\hline 4 & Q9UBJ2 & Peroxisomal farnesylated protein & 2 & 1.00 & $0.43 \pm 0.08$ & 0.21 & Miscellaneous function \\
\hline 5 & P37802 & Transgelin 2 & 2 & 1.00 & $0.56 \pm 0.01$ & 0.79 & Cytoskeletal protein- \\
\hline 6 & P67936 & Tropomyosin alpha 4 chain & 2 & 1.00 & $0.61 \pm 0.16$ & 0.83 & Cytoskeletal protein- \\
\hline 7 & Q5T0M7 & Fatty acid synthase & 2 & 1.00 & $0.65 \pm 0.17$ & $\mathrm{NA}^{d}$ & Synthase \\
\hline 8 & P49327 & Fatty acid synthase & 2 & 1.00 & $0.65 \pm 0.17$ & 0.48 & Synthase and synthetase \\
\hline 9 & O00151 & PDZ and LIM domain protein 1 & 1 & 0.99 & $0.67 \pm 0.78$ & 0.17 & Cytoskeletal protein- \\
\hline 10 & Q16850 & Cytochrome P450 51Al & 1 & 0.92 & $0.67 \pm 0.08$ & 1.24 & Oxidoreductase \\
\hline 11 & P04083 & Annexin 1 & 4 & 1.00 & $0.68 \pm 0.07$ & 1.02 & Select calcium binding protein \\
\hline 12 & P21964 & Catechol $O$-methyltransferase & 2 & 1.00 & $1.35 \pm 0.55$ & 0.81 & Transferase \\
\hline 13 & Q08257 & Quinone oxidoreductase & 1 & 1.00 & $1.40 \pm 0.28$ & 10.73 & Oxidoreductase \\
\hline 14 & P36969 & Phospholipid hydroperoxide glutathione peroxidase & 1 & 0.99 & $1.56 \pm 0.55$ & 1.14 & Oxidoreductase \\
\hline 15 & Q9H845 & Acyl-CoA dehydrogenase family member 9 & 1 & 1.00 & $1.58 \pm 0.77$ & $\mathrm{NA}^{d}$ & Oxidoreductase \\
\hline 16 & Q15738 & NAD(P)-dependent steroid dehydrogenase & 2 & 1.00 & $1.92 \pm 0.24$ & $\mathrm{NA}^{d}$ & Oxidoreductase \\
\hline 17 & Q01469 & Fatty acid-binding protein & 2 & 1.00 & $2.45 \pm 0.21$ & 1.67 & Transfer/carrier protein \\
\hline 18 & Q99714 & 3-hydroxyacyl-CoA dehydrogenase type II & 2 & 1.00 & $2.51 \pm 0.80$ & 1.90 & Oxidoreductase \\
\hline \multicolumn{8}{|c|}{ Nucleic Acid Metabolism } \\
\hline 1 & Q7Z2W4 & Zinc-finger $\mathrm{CCCH}$ type antiviral protein 1 & 3 & 1.00 & $0.27 \pm 0.05$ & 2.51 & Molecular function unclassified \\
\hline 2 & Q13951 & Core-binding factor, beta subunit & 1 & 1.00 & $0.31 \pm 0.05$ & 0.40 & Transcription factor \\
\hline 3 & P48512 & Transcription initiation factor IIB & 1 & 1.00 & $0.39 \pm 0.11$ & 1.25 & Transcription factor \\
\hline 4 & P53384 & Nucleotide-binding protein 1 & 2 & 1.00 & $0.44 \pm 0.05$ & 0.40 & Nucleic acid binding \\
\hline 5 & Q8TEK0 & FLJ00195 protein (Fragment) & 1 & 0.98 & $0.46 \pm 0.07$ & 0.31 & Transcription factor \\
\hline 6 & P18583 & SON protein & 1 & 0.98 & $0.47 \pm 0.11$ & 0.86 & Nucleic acid binding \\
\hline 7 & Q8N1F7 & Hypothetical protein KIAA0095 & 1 & 1.00 & $0.52 \pm 0.16$ & $\mathrm{NA}^{d}$ & Nucleic acid binding \\
\hline 8 & P78527 & DNA-dependent protein kinase catalytic subunit & 7 & 1.00 & $0.55 \pm 0.16$ & 0.41 & Kinase \\
\hline
\end{tabular}


Table I (Continued)

\begin{tabular}{|c|c|c|c|c|c|c|c|}
\hline & UniProt & protein name & $\begin{array}{l}\text { peptide } \\
\text { number }\end{array}$ & prob. ${ }^{a}$ & $\operatorname{cICAT}^{b}$ & Affymetrix $^{c}$ & molecular function \\
\hline \multicolumn{8}{|c|}{ Nucleic Acid Metabolism } \\
\hline 9 & Q12769 & Nuclear pore complex protein Nup160 & 1 & 0.94 & $0.55 \pm 0.11$ & 1.02 & transporter \\
\hline 10 & O75534 & UNR protein & 2 & 1.00 & $0.56 \pm 0.16$ & 0.98 & Nucleic acid binding \\
\hline 11 & P33991 & MCM4 minichromosome maintenance deficient 4 & 2 & 1.00 & $0.56 \pm 0.07$ & 0.18 & Nucleic acid binding \\
\hline 12 & P33992 & DNA replication licensing factor MCM5 & 3 & 1.00 & $0.58 \pm 0.25$ & 0.15 & Nucleic acid binding \\
\hline 13 & Q96EI0 & Similar to KIAA0801 gene product & 1 & 0.99 & $0.58 \pm 0.28$ & $\mathrm{NA}^{d}$ & Nucleic acid binding \\
\hline 14 & Q16576 & Histone acetyltransferase type B subunit 2 & 1 & 1.00 & $0.58 \pm 0.11$ & 0.54 & Miscellaneous function \\
\hline 15 & Q86VP6 & TIP120 protein & 3 & 1.00 & $0.58 \pm 0.04$ & $\mathrm{NA}^{d}$ & Transcription factor \\
\hline 16 & Q92878 & RAD50 & 1 & 0.96 & $0.58 \pm 0.18$ & $\mathrm{NA}^{d}$ & Nucleic acid binding \\
\hline 17 & P17812 & CTP synthase & 4 & 1.00 & $0.59 \pm 0.03$ & 0.81 & Synthase and synthetase \\
\hline 18 & O75766 & TRIP protein & 3 & 1.00 & $0.59 \pm 0.14$ & 0.92 & Transcription factor \\
\hline 19 & Q96KR1 & Putative zinc finger protein & 2 & 1.00 & $0.59 \pm 0.09$ & 1.04 & Nucleic acid binding \\
\hline 20 & Q9P0L0 & Vesicle-associated membrane protein-associated protein A & 2 & 1.00 & $0.59 \pm 0.17$ & 0.83 & Synthase and synthetase \\
\hline 21 & Q00839 & Heterogeneous nuclear ribonucleoprotein $\mathrm{U}$ & 6 & 1.00 & $0.60 \pm 0.20$ & 1.13 & Nucleic acid binding \\
\hline 22 & Q15097 & Polyadenylate-binding protein 2 & 3 & 1.00 & $0.64 \pm 0.09$ & 1.33 & Nucleic acid binding \\
\hline 23 & P11518 & 60 S ribosomal protein $\mathrm{L} 7 \mathrm{a}$ & 3 & 1.00 & $0.67 \pm 0.09$ & 1.26 & Nucleic acid binding \\
\hline 24 & P82979 & Nuclear protein Hcc-1 & 1 & 0.96 & $0.67 \pm 0.05$ & $\mathrm{NA}^{d}$ & Nucleic acid binding \\
\hline 25 & Q08945 & Structure-specific recognition protein 1 & 4 & 1.00 & $0.68 \pm 0.14$ & 1.19 & Transcription factor \\
\hline 26 & Q9NXD9 & Hypothetical protein FLJ20303 & 2 & 1.00 & $0.68 \pm 0.29$ & $\mathrm{NA}^{d}$ & Nucleic acid binding \\
\hline 27 & $\mathrm{O} 43172$ & U4/U6 small nuclear ribonucleoprotein Prp4 & 2 & 1.00 & $0.69 \pm 0.29$ & 0.55 & Nucleic acid binding \\
\hline 28 & P26358 & DNA (cytosine-5)-methyltransferase 1 & 1 & 0.99 & $0.69 \pm 0.04$ & 0.70 & Nucleic acid binding \\
\hline 29 & P26368 & Splicing factor U2AF 65 kDa subunit & 2 & 1.00 & $0.69 \pm 0.06$ & 1.26 & Nucleic acid binding \\
\hline 30 & Q09161 & 80 kDa nuclear cap binding protein & 3 & 1.00 & $1.32 \pm 0.52$ & 1.73 & Nucleic acid binding \\
\hline 31 & P14866 & Heterogeneous nuclear ribonucleoprotein L & 2 & 1.00 & $1.33 \pm 0.29$ & 1.15 & Nucleic acid binding \\
\hline 32 & P48634 & Hypothetical protein (Fragment) & 2 & 1.00 & $1.33 \pm 0.13$ & 1.19 & Nucleic acid binding \\
\hline 33 & Q13242 & Splicing factor, arginine/serine-rich 9 & 2 & 1.00 & $1.33 \pm 0.22$ & 0.75 & Nucleic acid binding \\
\hline 34 & Q9NQT4 & Exosome complex exonuclease RRP46 & 1 & 0.97 & $1.36 \pm 0.13$ & $\mathrm{NA}^{d}$ & Nucleic acid binding \\
\hline 35 & Q9GZT3 & DC50 (DC23) & 2 & 1.00 & $1.41 \pm 0.26$ & $\mathrm{NA}^{d}$ & Nucleic acid binding \\
\hline 36 & P31949 & Calgizzarin & 2 & 1.00 & $1.44 \pm 0.19$ & 2.06 & Select calcium binding protein \\
\hline 37 & P33240 & Cleavage stimulation factor, $64 \mathrm{kDa}$ subunit & 1 & 0.90 & $1.44 \pm 0.25$ & 0.73 & Nucleic acid binding \\
\hline 38 & Q9UKK9 & ADP-sugar pyrophosphatase YSAlH & 1 & 1.00 & $1.44 \pm 0.21$ & $\mathrm{NA}^{d}$ & Hydrolase \\
\hline 39 & P13489 & Placental ribonuclease inhibitor & 6 & 1.00 & $1.45 \pm 0.24$ & 1.02 & Select regulatory molecule \\
\hline 40 & P49321 & Nuclear autoantigenic sperm protein & 2 & 1.00 & $1.50 \pm 0.34$ & 0.77 & transporter \\
\hline 41 & Q96B26 & U6 snRNA-associated Sm-like protein LSm7 & 1 & 0.99 & $1.52 \pm 0.20$ & 3.41 & Nucleic acid binding \\
\hline 42 & P33316 & Deoxyuridine 5'-triphosphate nucleotidohydrolase & 3 & 1.00 & $1.54 \pm 0.21$ & 1.14 & Phosphatase \\
\hline 43 & P12956 & ATP-dependent DNA helicase II, 70 kDa subunit & 2 & 1.00 & $1.61 \pm 0.20$ & 0.81 & Nucleic acid binding \\
\hline 44 & Q96P11 & NOL1R & 1 & 1.00 & $1.61 \pm 0.32$ & $\mathrm{NA}^{d}$ & Nucleic acid binding \\
\hline 45 & P55795 & Heterogeneous nuclear ribonucleoprotein $\mathrm{H}^{\prime}$ & 1 & 0.99 & $1.62 \pm 0.41$ & 1.12 & Nucleic acid binding \\
\hline 46 & Q01081 & Splicing factor U2AF $35 \mathrm{kDa}$ subuni & 1 & 0.99 & $1.63 \pm 0.24$ & 1.29 & Nucleic acid binding \\
\hline 47 & P49915 & GMP synthase [glutamine-hydrolyzing] & 2 & 0.98 & $1.71 \pm 0.34$ & 1.23 & Synthase \\
\hline 48 & P05455 & Lupus La protein & 1 & 1.00 & $1.82 \pm 0.10$ & 1.51 & Nucleic acid binding \\
\hline 49 & Q9BV68 & Hypothetical protein & 2 & 0.93 & $1.82 \pm 0.28$ & 1.35 & Nucleic acid binding \\
\hline 50 & Q9Y3A5 & Shwachman-Bodian-Diamond syndrome protein & 1 & 1.00 & $1.83 \pm 0.30$ & $\mathrm{NA}^{d}$ & Molecular function unclassified \\
\hline 51 & P55769 & NHP2-like protein 1 & 2 & 1.00 & $1.86 \pm 0.69$ & 2.01 & Nucleic acid binding \\
\hline 52 & P09429 & High mobility group protein 1 & 5 & 1.00 & $1.92 \pm 0.24$ & 1.25 & Transcription factor \\
\hline 53 & P18754 & Regulator of chromosome condensation & 1 & 0.99 & $1.92 \pm 0.25$ & 0.87 & Nucleic acid binding \\
\hline 54 & P31939 & Bifunctional purine biosynthesis protein PURH & 4 & 1.00 & $2.07 \pm 0.64$ & 2.21 & Hydrolase \\
\hline 55 & Q9Y265 & RuvB-like 1 & 1 & 0.99 & $2.03 \pm 0.38$ & 0.70 & Nucleic acid binding \\
\hline 56 & Q16531 & DNA damage binding protein 1 & 1 & 0.93 & $2.26 \pm 0.13$ & 1.25 & Nucleic acid binding \\
\hline 57 & Q15365 & Poly(rC)-binding protein 1 & 5 & 1.00 & $2.29 \pm 0.34$ & 1.38 & Nucleic acid binding \\
\hline 58 & P00492 & Hypoxanthine-guanine phosphoribosyltransferase & 4 & 1.00 & $2.67 \pm 0.19$ & 1.25 & Transferase \\
\hline 59 & Q9HCU5 & Prolactin regulatory element-binding protein & 1 & 0.98 & $3.24 \pm 0.29$ & 5.10 & Nucleic acid binding \\
\hline 60 & P36959 & GMP reductase 1 & 1 & 0.99 & $33.64 \pm 6.76$ & 1.57 & Oxidoreductase \\
\hline \multicolumn{8}{|c|}{ Oncogenesis } \\
\hline 1 & P08729 & Keratin, type II cytoskeletal 7 & 1 & 1.00 & $0.00 \pm 0.00$ & 0.02 & Select regulatory molecule \\
\hline 2 & Q9BYX2 & $\mathrm{TBC} 1$ domain family member 2 & 1 & 0.96 & $0.13 \pm 0.03$ & $\mathrm{NA}^{d}$ & Select regulatory molecule \\
\hline 3 & Q06136 & Follicular variant translocation protein 1 precursor & 1 & 1.00 & $0.31 \pm 0.05$ & 0.43 & Oxidoreductase \\
\hline 4 & P57764 & DFN5-like protein FLJ12150 & 1 & 1.00 & $0.33 \pm 0.24$ & $\mathrm{NA}^{d}$ & Molecular function unclassified \\
\hline 5 & P13797 & T-plastin & 4 & 1.00 & $0.34 \pm 0.21$ & 0.33 & Cytoskeletal protein \\
\hline 6 & P46013 & Antigen KI-67 & 3 & 1.00 & $0.52 \pm 0.05$ & 0.49 & Miscellaneous function \\
\hline 7 & P18206 & Vinculin & 4 & 1.00 & $0.54 \pm 0.27$ & 0.40 & Cytoskeletal protein \\
\hline 8 & Q92688 & Acidic leucine-rich nuclear phosphoprotein 32 family member B & 2 & 1.00 & $1.66 \pm 0.16$ & 1.01 & Select regulatory molecule \\
\hline 9 & P39687 & Acidic leucine-rich nuclear phosphoprotein 32 family member A & 2 & 0.99 & $1.67 \pm 0.41$ & 1.40 & Select regulatory molecule \\
\hline \multicolumn{8}{|c|}{ Other Metabolism } \\
\hline 1 & Q16881 & Thioredoxin reductase, cytoplasmic precursor & 1 & 0.93 & $0.6 \pm 0.20$ & 0.56 & Oxidoreductase \\
\hline 2 & Q04760 & Lactoylglutathione lyase & 2 & 1.00 & $1.31 \pm 0.20$ & 1.11 & Lyase \\
\hline 3 & P49189 & Aldehyde dehydrogenase, E3 isozyme & 1 & 0.99 & $1.44 \pm 0.86$ & 0.65 & Oxidoreductase \\
\hline 4 & P10599 & Thioredoxin & 3 & 1.00 & $1.63 \pm 0.10$ & 2.11 & Oxidoreductase \\
\hline 5 & Q8UBL6 & Uroporphyrinogen decarboxylase & 1 & 1.00 & $1.99 \pm 0.27$ & 0.98 & Lyase \\
\hline 6 & Q9BW04 & Hypothetical protein FLJ36507 & 1 & 1.00 & $6.72 \pm 0.92$ & $\mathrm{NA}^{d}$ & Select regulatory molecule \\
\hline
\end{tabular}


Table I (Continued)

\begin{tabular}{|c|c|c|c|c|c|c|c|}
\hline & UniProt & protein name & $\begin{array}{l}\text { peptide } \\
\text { number }\end{array}$ & prob. ${ }^{a}$ & $\operatorname{cICAT}^{b}$ & Affymetrix ${ }^{c}$ & molecular function \\
\hline \multicolumn{8}{|c|}{ Protein Metabolism and Modification } \\
\hline 1 & P21980 & Protein-glutamine gamma-glutamyltransferase & 1 & 0.97 & $0.00 \pm 0.00$ & $\mathrm{NA}^{d}$ & Transferase \\
\hline 2 & Q9UK22 & F-box only protein 2 & 2 & 1.00 & $0.14 \pm 0.04$ & 1.26 & Miscellaneous function \\
\hline 3 & Q86YM9 & Calpastatin (Fragment) & 1 & 1.00 & $0.25 \pm 0.22$ & 0.78 & Select regulatory molecule \\
\hline 4 & P48594 & Squamous cell carcinoma antigen 2 & 1 & 1.00 & $0.27 \pm 0.03$ & $\mathrm{NA}^{d}$ & Select regulatory molecule \\
\hline 5 & P63208 & S-phase kinase-associated protein $1 \mathrm{~A}$ & 1 & 0.97 & $0.35 \pm 0.05$ & 0.90 & Nucleic acid binding \\
\hline 6 & Q15418 & Ribosomal protein S6 kinase alpha 1 & 2 & 1.00 & $0.39 \pm 0.09$ & 0.27 & Kinase \\
\hline 7 & P15170 & G1 to $\mathrm{S}$ phase transition protein 1 homologue & 2 & 1.00 & $0.41 \pm 0.27$ & 0.60 & Nucleic acid binding \\
\hline 8 & $\mathrm{O} 43776$ & Asparaginyl-tRNA synthetase, cytoplasmic & 2 & 1.00 & $0.48 \pm 0.13$ & 0.96 & Nucleic acid binding \\
\hline 9 & P18282 & Destrin & 5 & 0.91 & $0.50 \pm 0.13$ & 0.93 & Cytoskeletal protein \\
\hline 10 & Q13564 & Amyloid protein-binding protein 1 & 2 & 1.00 & $0.50 \pm 0.05$ & 0.43 & Ligase \\
\hline 11 & P36578 & $60 \mathrm{~S}$ ribosomal protein $\mathrm{L} 4$ & 2 & 1.00 & $0.51 \pm 0.05$ & 1.86 & Nucleic acid binding \\
\hline 12 & Q16763 & Ubiquitin-conjugating enzyme E2-24 kDa & 1 & 0.98 & $0.54 \pm 0.07$ & 1.17 & ligase \\
\hline 13 & P13862 & Casein kinase II beta chain & 2 & 1.00 & $0.55 \pm 0.09$ & 1.84 & Kinase \\
\hline 14 & P04643 & $40 S$ ribosomal protein S11 & 3 & 1.00 & $0.59 \pm 0.05$ & 1.57 & Nucleic acid binding \\
\hline 15 & P04645 & 60 S ribosomal protein L30 & 2 & 1.00 & $0.60 \pm 0.03$ & 1.34 & Nucleic acid binding \\
\hline 16 & Q12765 & Y193_HUMAN Hypothetical protein KIAA0193 & 1 & 1.00 & $0.60 \pm 0.26$ & 1.13 & Select regulatory molecule \\
\hline 17 & P02433 & $60 S$ ribosomal protein $\mathrm{L} 32$ & 2 & 1.00 & $0.63 \pm 0.18$ & 1.11 & Nucleic acid binding \\
\hline 18 & P50914 & 60 S ribosomal protein $\mathrm{L} 14$ & 4 & 1.00 & $0.64 \pm 0.08$ & 2.35 & Nucleic acid binding \\
\hline 19 & P28838 & Cytosol aminopeptidase & 3 & 1.00 & $0.64 \pm 0.25$ & 0.95 & Protease \\
\hline 20 & Q92524 & $26 \mathrm{~S}$ protease regulatory subunit S10B & 2 & 1.00 & $0.65 \pm 0.11$ & 1.91 & Hydrolase \\
\hline 21 & P31689 & DnaJ homologue subfamily A member 1 & 3 & 1.00 & $0.67 \pm 0.52$ & 0.56 & Chaperone \\
\hline 22 & Q15070 & Cytochrome oxidase biogenesis protein OXA1, mitochondrial precursor & 2 & 1.00 & $0.67 \pm 0.09$ & 0.88 & Transporter \\
\hline 23 & Q93009 & Ubiquitin carboxyl-terminal hydrolase 7 & 1 & 1.00 & $0.67 \pm 0.13$ & 0.82 & Protease \\
\hline 24 & Q9Y5M8 & Signal recognition particle receptor beta subunit & 2 & 1.00 & $0.67 \pm 0.05$ & 0.96 & signaling molecule \\
\hline 25 & P08238 & Heat shock protein 90 beta & 4 & 1.00 & $0.68 \pm 0.21$ & 0.89 & Chaperone \\
\hline 26 & P49721 & Proteasome subunit beta type 2 & 3 & 1.00 & $0.68 \pm 0.11$ & 1.08 & Protease \\
\hline 27 & Q96AE4 & FUSE binding protein & 1 & 1.00 & $0.69 \pm 0.12$ & 0.79 & Nucleic acid binding \\
\hline 28 & Q8N425 & Likely ortholog of mouse ubiquitin-conjugating enzyme E2-230K & 2 & 1.00 & $0.69 \pm 0.07$ & $\mathrm{NA}^{d}$ & ligase \\
\hline 29 & Q99543 & Zuotin related factor- 1 & 1 & 0.96 & $1.39 \pm 0.29$ & 3.39 & Chaperone \\
\hline 30 & P30405 & Peptidyl-prolyl cis-trans isomerase, mitochondrial precursor & 1 & 1.00 & $1.40 \pm 0.29$ & 1.62 & Isomerase \\
\hline 31 & Q02790 & FK506-binding protein 4 & 2 & 1.00 & $1.46 \pm 0.15$ & 1.17 & Isomerase \\
\hline 32 & Q9NR50 & Translation initiation factor eIF-2B gamma subunit & 1 & 0.99 & $1.48 \pm 0.35$ & 0.90 & Nucleic acid binding \\
\hline 33 & P49591 & Seryl-tRNA synthetase & 1 & 0.99 & $1.49 \pm 0.41$ & 2.87 & Nucleic acid binding \\
\hline 34 & P36776 & Lon protease homolog, mitochondrial precursor & 1 & 1.00 & $1.57 \pm 0.41$ & 1.25 & protease \\
\hline 35 & Q92598 & Heat-shock protein $105 \mathrm{kDa}$ & 7 & 1.00 & $1.60 \pm 0.17$ & 1.33 & Chaperone \\
\hline 36 & P07858 & Cathepsin B & 3 & 1.00 & $1.61 \pm 0.14$ & 2.16 & Protease \\
\hline 37 & P53634 & Cathepsin C & 1 & 1.00 & $1.62 \pm 0.36$ & 0.28 & Protease \\
\hline 38 & P34932 & Heat shock 70 kDa protein 4 & 3 & 1.00 & $1.67 \pm 0.26$ & 2.24 & Chaperone \\
\hline 39 & P35998 & $26 \mathrm{~S}$ protease regulatory subunit 7 & 2 & 1.00 & $1.69 \pm 0.51$ & 2.37 & Hydrolase \\
\hline 40 & P14868 & Aspartyl-tRNA synthetase & 1 & 0.96 & $1.73 \pm 1.30$ & 2.36 & Nucleic acid binding \\
\hline 41 & P62276 & 40 S ribosomal protein S29 & 1 & 1.00 & $1.74 \pm 0.32$ & 1.59 & Nucleic acid binding \\
\hline 42 & Q13404 & DNA-binding protein & 1 & 0.94 & $1.74 \pm 0.06$ & $\mathrm{NA}^{d}$ & Ligase \\
\hline 43 & Q99538 & Legumain precursor & 1 & 0.97 & $1.77 \pm 0.30$ & $\mathrm{NA}^{d}$ & Protease \\
\hline 44 & Q9UBT2 & ANTHRACYCLINE-associated resistance ARX & 3 & 1.00 & $1.86 \pm 0.23$ & 2.19 & Ligase \\
\hline 45 & Q15765 & Prefoldin subunit 3 & 2 & 1.00 & $1.87 \pm 0.43$ & 3.08 & transporter \\
\hline 46 & P27924 & Ubiquitin-conjugating enzyme E2-25 kDa & 1 & 0.96 & $1.95 \pm 0.23$ & 1.60 & Ligase \\
\hline 47 & Q92507 & ES1 protein homologue, mitochondrial precursor & 1 & 1.00 & $2.28 \pm 0.24$ & 110.10 & Miscellaneous function \\
\hline 48 & P25685 & DnaJ homologue subfamily B member 1 & 2 & 1.00 & $2.46 \pm 0.41$ & 1.58 & Chaperone \\
\hline 49 & P29144 & Tripeptidyl-peptidase II & 4 & 1.00 & $3.01 \pm 1.10$ & 2.92 & Hydrolase \\
\hline 50 & P41250 & Glycyl-tRNA synthetase & 1 & 1.00 & $6.8 \pm 2.66$ & 2.79 & Synthase and synthetase \\
\hline \multicolumn{8}{|c|}{ Signal Transduction and Regulation } \\
\hline 1 & Q86ST5 & Similar to centaurin, gamma 3 & 1 & 0.99 & $0.00 \pm 0.00$ & $\mathrm{NA}^{d}$ & GTP binding \\
\hline 2 & Q9UBI6 & Guanine nucleotide-binding protein $\mathrm{G}(\mathrm{I}) / \mathrm{G}(\mathrm{S}) / \mathrm{G}(\mathrm{O})$ gamma-12 subunit & 2 & 1.00 & $0.20 \pm 0.04$ & 0.58 & Select regulatory molecule \\
\hline 3 & P35613 & Basigin precursor & 2 & 1.00 & $0.30 \pm 0.03$ & 0.56 & Signaling molecule \\
\hline 4 & P06749 & Transforming protein RhoA & 4 & 1.00 & $0.35 \pm 0.22$ & 1.13 & Select regulatory molecule \\
\hline 5 & P05362 & Intercellular adhesion molecule-1 precursor & 2 & 1.00 & $0.38 \pm 0.05$ & $\mathrm{NA}^{d}$ & Cell adhesion molecule \\
\hline 6 & Q13578 & DNA repair protein & 1 & 1.00 & $0.41 \pm 0.07$ & $\mathrm{NA}^{d}$ & Phosphatase \\
\hline 7 & P30086 & Phosphatidylethanolamine-binding protein & 1 & 0.98 & $0.48 \pm 0.09$ & 0.62 & Select regulatory molecule \\
\hline 8 & P08134 & Transforming protein RhoC & 2 & 0.99 & $0.54 \pm 0.13$ & 0.58 & Select regulatory molecule \\
\hline 9 & P84097 & Rho-related GTP-binding protein RhoG & 1 & 1.00 & $0.55 \pm 0.13$ & 0.83 & Select regulatory molecule \\
\hline 10 & Q9Y572 & Receptor-interacting serine/threonine protein kinase 3 & 1 & 0.91 & $0.58 \pm 0.05$ & $\mathrm{NA}^{d}$ & Kinase \\
\hline 11 & P43490 & Pre-B cell enhancing factor precursor & 2 & 1.00 & $0.65 \pm 0.09$ & 1.46 & Signaling molecule \\
\hline 12 & P60953 & Cell division control protein 42 homologue & 3 & 1.00 & $0.67 \pm 0.20$ & 0.88 & Select regulatory molecule \\
\hline 13 & P04901 & Guanine nucleotide-binding protein $\mathrm{G}(\mathrm{I}) / \mathrm{G}(\mathrm{S}) / \mathrm{G}(\mathrm{T})$ beta subunit 1 & 1 & 1.00 & $0.68 \pm 0.05$ & 0.70 & Select regulatory molecule \\
\hline 14 & P04899 & Guanine nucleotide-binding protein G(i), alpha-2 subunit & 1 & 1.00 & $1.32 \pm 0.24$ & 0.44 & Select regulatory molecule \\
\hline 15 & Q9HCY8 & S100 calcium-binding protein Al4 & 2 & 1.00 & $1.41 \pm 0.19$ & 0.36 & Select calcium binding protein \\
\hline 16 & P29312 & 14-3-3 protein zeta/delta & 3 & 1.00 & $1.48 \pm 0.12$ & 1.40 & Select regulatory molecule \\
\hline
\end{tabular}


Table I (Continued)

\begin{tabular}{|c|c|c|c|c|c|c|c|}
\hline & UniProt & protein name & $\begin{array}{l}\text { peptide } \\
\text { number }\end{array}$ & prob. $^{a}$ & $\mathrm{cICAT}^{b}$ & Affymetrix $^{c}$ & molecular function \\
\hline \multicolumn{8}{|c|}{ Signal Transduction and Regulation } \\
\hline 17 & Q12797 & Aspartyl/asparaginyl beta-hydroxylase & 1 & 0.91 & $1.69 \pm 0.34$ & 1.09 & Oxidoreductase \\
\hline 18 & O60716 & Catenin, $120 \mathrm{ctn}$ & 1 & 1.00 & $1.78 \pm 0.34$ & 1.16 & Cell junction protein \\
\hline 19 & P16144 & Integrin beta- 4 & 2 & 1.00 & $1.96 \pm 0.96$ & 1.91 & Cell adhesion molecule \\
\hline 20 & P01116 & Transforming protein $\mathrm{p} 21 \mathrm{~A}$ & 1 & 0.98 & $2.07 \pm 0.49$ & 2.78 & Select regulatory molecule \\
\hline 21 & P16422 & Tumor-associated calcium signal transducer 1 precursor & 1 & 0.99 & $2.12 \pm 0.43$ & 1.49 & Signaling molecule \\
\hline 22 & Q86VB7 & Similar to CD163 antigen & 1 & 0.95 & $2.18 \pm 0.46$ & $\mathrm{NA}^{d}$ & Receptor \\
\hline 23 & P31947 & $14-3-3$ protein sigma & 4 & 1.00 & $2.45 \pm 0.17$ & 1.79 & Select regulatory molecule \\
\hline 24 & P27348 & $14-3-3$ protein tau & 3 & 1.00 & $2.51 \pm 0.24$ & 1.56 & Select regulatory molecule \\
\hline 25 & P23229 & Integrin alpha-6 & 1 & 0.99 & $3.34 \pm 1.06$ & 1.81 & Cell adhesion molecule \\
\hline 26 & P35222 & Catenin, beta & 4 & 1.00 & $5.89 \pm 1.3$ & 1.91 & Signaling molecule \\
\hline 27 & O95996 & APCL protein & 1 & 0.99 & $8.37 \pm 0.35$ & $\mathrm{NA}^{d}$ & Cytoskeletal protein \\
\hline 28 & Q8IXW0 & Hypothetical protein & 1 & 0.94 & $9999 \pm 0.00$ & $\mathrm{NA}^{d}$ & Signaling molecule \\
\hline \multicolumn{8}{|c|}{ Transport } \\
\hline 1 & P16615 & Sarcoplasmic/endoplasmic reticulum calcium ATPase 2 & 2 & 1.00 & $0.64 \pm 0.16$ & 0.50 & Transporter \\
\hline 2 & P35658 & Nuclear pore complex protein Nup214 & 2 & 1.00 & $0.69 \pm 0.08$ & $\mathrm{NA}^{d}$ & Transporter \\
\hline 3 & P45880 & Voltage-dependent anion-selective channel protein 2 & 1 & 1.00 & $0.69 \pm 0.13$ & 1.34 & Ion channel \\
\hline 4 & O00299 & Chloride intracellular channel protein 1 & 4 & 1.00 & $2.38 \pm 0.63$ & 1.99 & Ion channel \\
\hline \multicolumn{8}{|c|}{ Biological Process Unclassified } \\
\hline 1 & Q9H690 & Hypothetical protein FLJ22484 & 2 & 1.00 & $0.00 \pm 0.00$ & $\mathrm{NA}^{d}$ & Molecular function unclassified \\
\hline 2 & Q9BSE0 & Hypothetical protein & 1 & 0.94 & $0.13 \pm 0.01$ & $\mathrm{NA}^{d}$ & Molecular function unclassified \\
\hline 3 & Q8N8W6 & Hypothetical protein FLJ38753 & 2 & 1.00 & $0.24 \pm 0.08$ & $\mathrm{NA}^{d}$ & Molecular function unclassified \\
\hline 4 & Q96G34 & DC12 protein & 1 & 0.91 & $0.37 \pm 0.09$ & $\mathrm{NA}^{d}$ & Molecular function unclassified \\
\hline 5 & Q9BTE3 & Hypothetical protein FLJ13081 & 2 & 1.00 & $0.50 \pm 0.05$ & $\mathrm{NA}^{d}$ & Molecular function unclassified \\
\hline 6 & Q96FT1 & Hypothetical protein (Fragment) & 1 & 0.98 & $0.50 \pm 0.08$ & $\mathrm{NA}^{d}$ & Nucleic acid binding \\
\hline 7 & Q86U38 & Similar to RIKEN cDNA 2610027L16 gene & 1 & 1.00 & $0.56 \pm 0.07$ & $\mathrm{NA}^{d}$ & Molecular function unclassified \\
\hline 8 & Q9BQ69 & Protein LRP16 & 2 & 1.00 & $0.56 \pm 0.22$ & $\mathrm{NA}^{d}$ & transporter \\
\hline 9 & Q9Y2L0 & KIAA1007 protein (Fragment) & 2 & 1.00 & $0.59 \pm 0.08$ & $\mathrm{NA}^{d}$ & Molecular function unclassified \\
\hline 10 & Q9Y4W6 & AFG3-like protein 2 & 4 & 1.00 & $0.63 \pm 0.12$ & 0.81 & protease \\
\hline 11 & Q14166 & Hypothetical protein KIAA0153 & 2 & 0.98 & $0.65 \pm 0.04$ & 1.30 & Molecular function unclassified \\
\hline 12 & Q13826 & Autoantigen & 2 & 1.00 & $0.69 \pm 0.14$ & $\mathrm{NA}^{d}$ & protein binding \\
\hline 13 & P42704 & $130 \mathrm{kDa}$ leucine-rich protein & 2 & 1.00 & $1.54 \pm 0.17$ & 1.32 & Nucleic acid binding \\
\hline 14 & Q8N3B3 & Hypothetical protein (Fragment) & 2 & 1.00 & $1.57 \pm 0.25$ & $\mathrm{NA}^{d}$ & Molecular function unclassified \\
\hline 15 & Q9H3K6 & My016 protein & 1 & 0.95 & $1.91 \pm 0.41$ & 3.44 & Molecular function unclassified \\
\hline 16 & O75223 & Hypothetical protein & 2 & 1.00 & $2.2 \pm 0.23$ & 2.60 & Molecular function unclassified \\
\hline 17 & Q9H4L5 & Oxysterol binding protein-related protein 3 & 1 & 1.00 & $3.06 \pm 5.19$ & 3.73 & Protein binding \\
\hline 18 & Q810P0 & similar to testis expressed sequence $13 \mathrm{~A}$ & 1 & 1.00 & $7.51 \pm 9.10$ & $\mathrm{NA}^{d}$ & Molecular function unclassified \\
\hline 19 & Q9H6W2 & Hypothetical protein FLJ21810 & 1 & 0.98 & $11.04 \pm 2.88$ & $\mathrm{NA}^{d}$ & Molecular function unclassified \\
\hline
\end{tabular}

${ }^{a}$ ProteinProphet score. ${ }^{b}$ The fold change in the protein ratio of TMC-1/SC-M1 cells. ${ }^{c}$ The fold change in the mRNA ratio of TMC-1/SC-M1 cells. ${ }^{d}$ ND, not available.

of microarray and proteomic results were performed by their corresponding gene names. Among the 240 differentially expressed proteins, 226 of the proteins found by cICAT analysis could be matched to their corresponding mRNA expression levels. In the correlation scattergram shown in Figure 4, proteins for which mRNA fold changes have a positive correlation with protein level changes appear on or near the $45^{\circ}$ diagonal. The majority of differentially expressed mRNAs and cognate proteins correlated positively with a statistically significant correlation coefficient of 0.29 . In TMC- 1 cells, 29 of the 226 proteins with elevated mRNA levels were found to be at least overexpressed by 1.3-fold at the protein level. Likewise, 38 TMC-1 cell proteins showed a correlation between reduced protein levels and reduced mRNA levels. However, the result also revealed that some proteins are not transcriptionally regulated or show opposite regulation patterns. As shown in Figure 4, seven proteins showed a negative correlation between protein and mRNA expression levels. This is in accordance with the conclusion that noncell-structure-related proteins show poor correlation between mRNA and protein levels. ${ }^{30}$

Validation of Candidate Genes That Are Differentially Expressed in SC-M1 and TMC-1 Cells. To further evaluate the results obtained from the global comparative expression studies, we examined the expression status of several candidate genes identified by comparative proteomics in the SC-M1 and TMC- 1 cells. $\beta$-Catenin, galectin 1, IQ motif-containing GTPase activating protein (IQGAP1), vimentin, integrin $\alpha 6$, and integrin $\beta 4$ were chosen because they have been previously shown to be involved in the pathogenic development of tumor formation and progression, and in particular, some are implicated in the process of acquiring metastatic capability in various cancers (Table 3). Semiquantitative RT-PCR analysis was performed to examine the transcript levels of these genes. In agreement with the proteomic and genomic findings, higher levels of $\beta$-catenin, galectin 1 , vimentin, integrin $\alpha 6$, and integrin $\beta 4$ transcripts were observed in the metastatic TMC-1 cells, whereas IQGAP1 was expressed at a lower level in TMC-1 cells (Figure 5A). Integrins are the major cell surface receptors involved in cellcell attachment and cell migration. The flow cytometry analysis showed significantly higher levels of integrin $\alpha 6$ and $\beta 4$ were localized at the cell surface of metastatic TMC-1 cells than SCM1 cells (Figure 5B). $\beta$-Catenin plays dual roles in adhesion and transducer/transcriptional regulaton. ${ }^{31}$ When the Wnt signal is activated, $\beta$-catenin is not phosphorylated and accumulates in the cytoplasm, leading to the subsequent nuclear translocation..$^{32}$ Therefore, we examined the expression status of $\beta$-catenin by immunocytostaining. As shown in Figure 5C, $\beta$-catenin was abundantly expressed and accumulated in the 


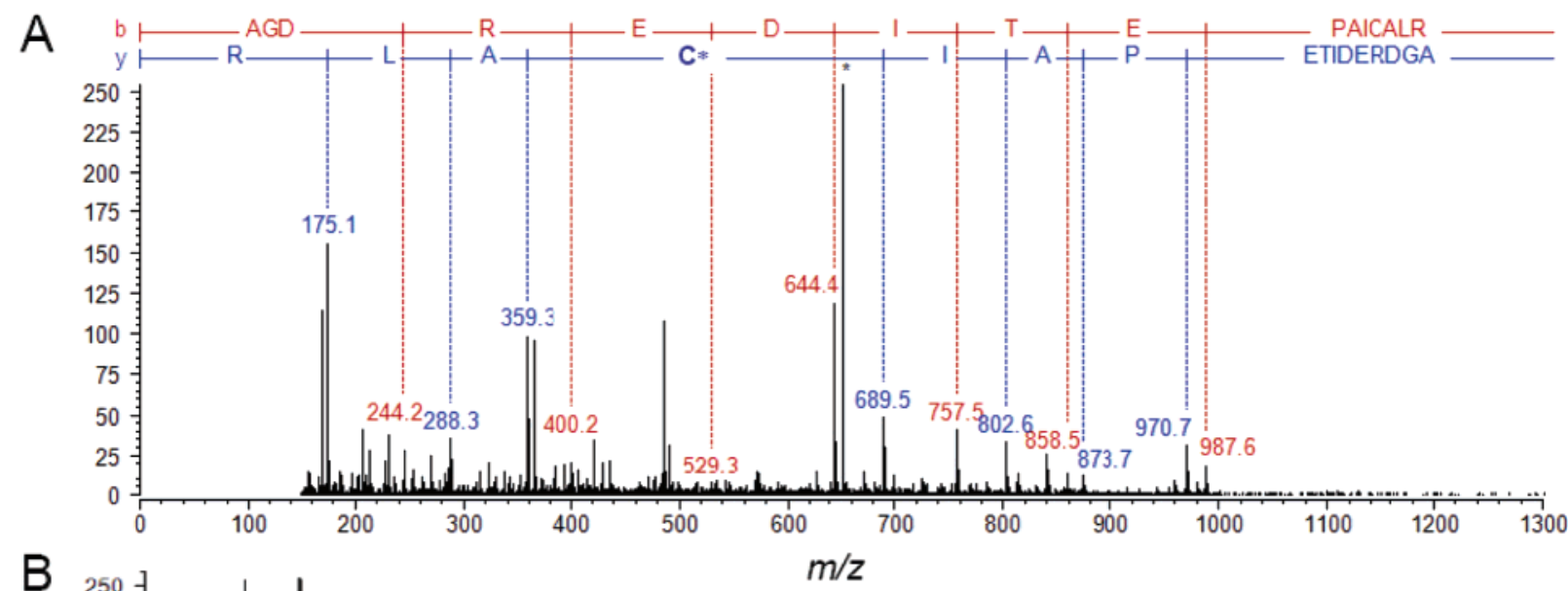

B

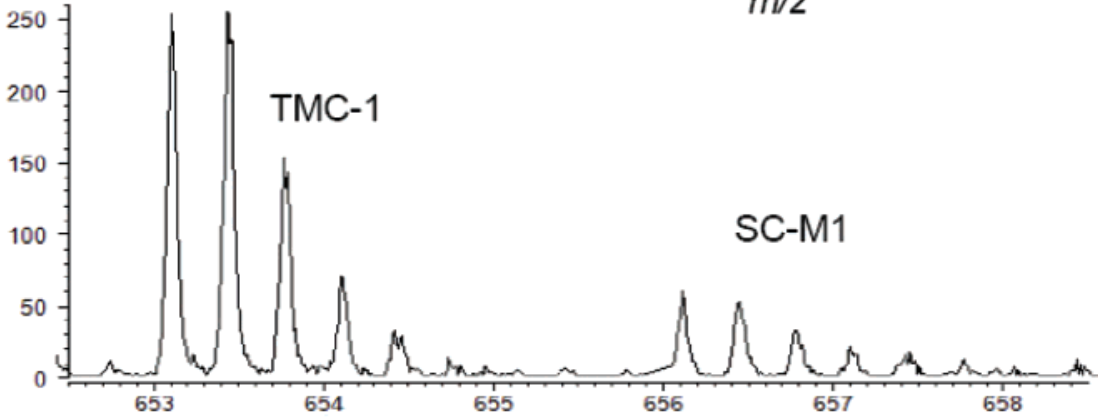

\section{SC-M1 TMC-1}

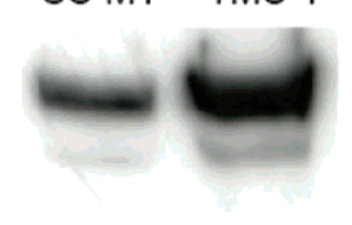

$\beta$-catenin
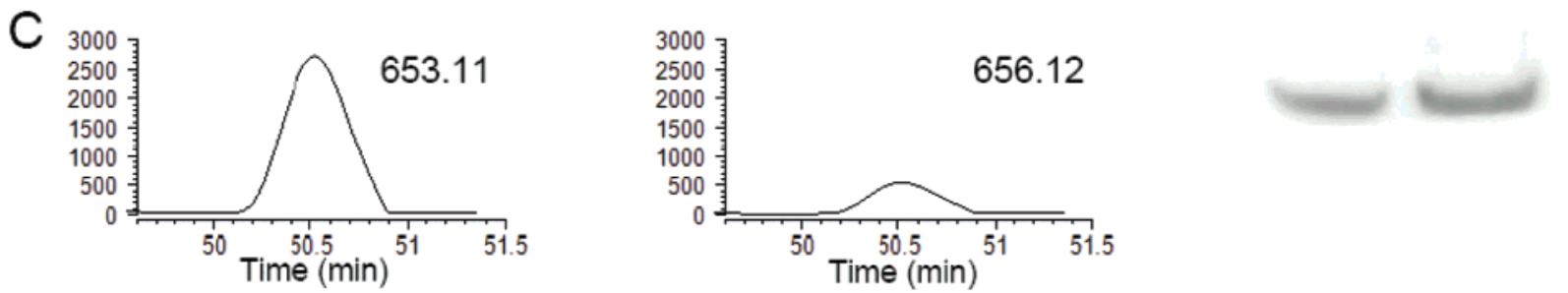

Actin

Figure 3. Quantitation and confirmation of differential expression of $\beta$-catenin in SC-M1 and TMC-1 cells. The MS/MS spectrum (A) and MS spectrum (B) of the light (TMC-1) and heavy (SC-M1) cICAT isotope-labeled peptide AGDREDITEPAICALR. (C) The corresponding extracted ion chromatogram revealed a 5.9 -fold overexpression of $\beta$-catenin in TMC- 1 cells. (D) Western blotting analysis confirmed that $\beta$-catenin was overexpressed in TMC-1 cells.

Table 2. Pathways Containing Genes Differentially Expressed between TMC-1 and SC-M1 Cells from Microarray Gene Expression Measurements

\begin{tabular}{|c|c|c|c|}
\hline pathway & $N^{a}$ & $T^{b}$ & $\begin{array}{l}\% \text { differentially } \\
\text { expressed genes }\end{array}$ \\
\hline Proepithelin Conversion to Epithelin and Wound Repair Control & 6 & 6 & 100 \\
\hline Inhibition of Matrix Metalloproteinases & 8 & 10 & 80 \\
\hline IL-18 Signaling Pathway & 7 & 9 & 78 \\
\hline OX40 Signaling Pathway & 5 & 7 & 71 \\
\hline Multidrug Resistance Factors & 5 & 7 & 71 \\
\hline Neuroregulin Receptor Degradation Protein-1 Controls ErbB3 Receptor Recycling & 9 & 13 & 69 \\
\hline Antigen-dependent B Cell Activation & 11 & 16 & 69 \\
\hline GATA3 Activation of the Th2 Cytokine Genes Expression & 10 & 15 & 67 \\
\hline CDK Regulation of DNA Replication & 6 & 9 & 67 \\
\hline Nitric Oxide Signaling Pathway & 6 & 9 & 67 \\
\hline Bystander B Cell Activation & 7 & 11 & 64 \\
\hline Platelet Amyloid Precursor Protein Pathway & 7 & 11 & 64 \\
\hline Sonic Hedgehog (Shh) Pathway & 7 & 11 & 64 \\
\hline TSP-1-Induced Apoptosis in Microvascular Endothelial Cells & 5 & 8 & 63 \\
\hline Vit-C in Brain & 5 & 8 & 63 \\
\hline METS Effect on Macrophage Differentiation & 12 & 20 & 60 \\
\hline FOSB Gene Expression and Drug Abuse & 3 & 5 & 60 \\
\hline
\end{tabular}

${ }^{a} N=$ number of differentially expressed genes in the pathway. ${ }^{b} T=$ the total number of probed genes in the pathway.

cytoplasm of TMC-1 cells, whereas it had a more restricted distribution in SC-M1 cells, mostly localized to the plasma membranes.
Because apoptosis dysfunction in metastases has been suggested to participate in their poor response to conventional anticancer treatments ${ }^{33}$ and because of our finding of dif- 


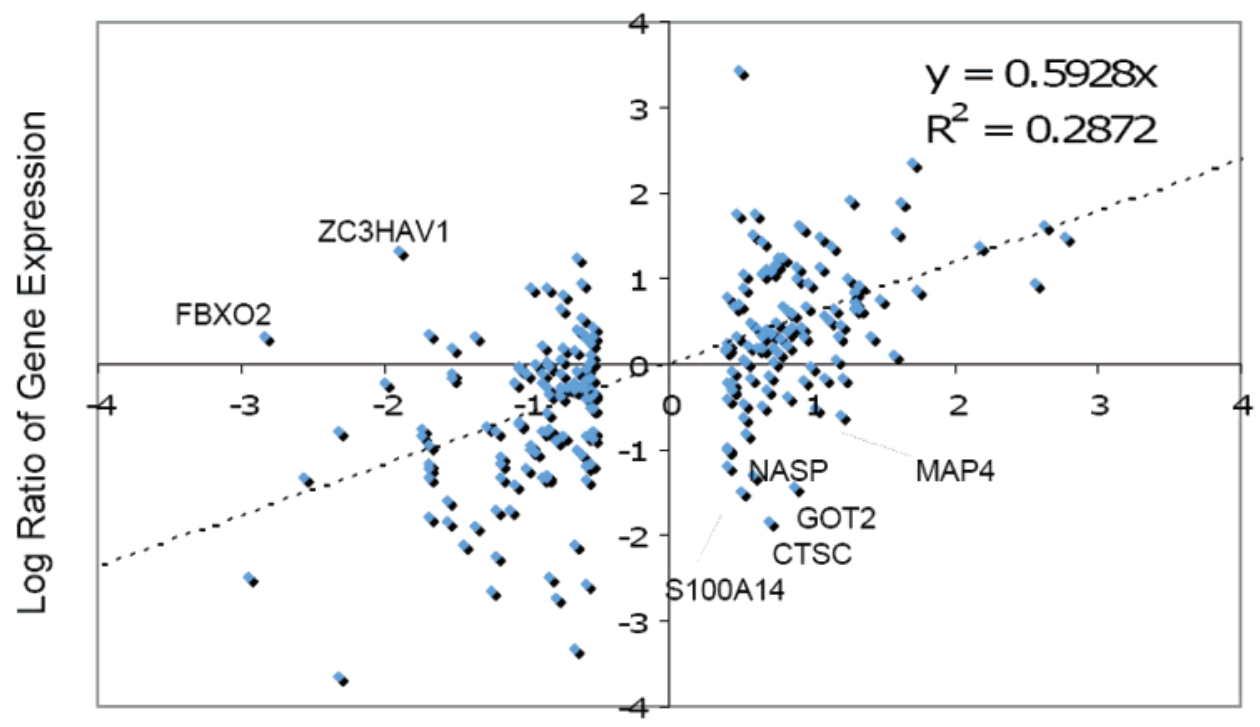

Log Ratio of Protein Expression

Figure 4. Scatter plot showing the protein ratios obtained by the cICAT analysis, and 2D-LC-MS/MS and mRNA ratios obtained from the Affymetrix DNA microarray for SC-M1 and TMC-1 cells. The labeled proteins showed a significant negative correlation between protein and mRNA expression levels. Both expression ratios are plotted in logarithmic scale.

ferential expression of proteins in the TSP-1-induced apoptosis pathway, we also examined whether SC-M1 and TMC-1 cells displayed different degrees of resistance toward chemotherapeutic drug-induced apoptosis. Cell viabilityies were measured after treatment with increasing concentrations of 5-fluorouracil. As shown in Figure 6, 5-fluorouracil increased cell death in a time- and dose-dependent manner in SC-M1 cells. By comparison, TMC-1 cells were more resistant to 5-fluorouracilinduced cell death.

\section{Discussion}

Comparison of Genomic and Proteomic Profiling. In this study, we compared the global protein and mRNA patterns of nonmetastatic SC-M1 and metastatic TMC-1 gastric cancer cells to elucidate the molecular changes associated with carcinogenesis and metastasis. As shown in Tables 1 and 2, the functional classification of the differentially expressed proteins and mRNAs indicates that the DNA microarray and cICAT technique have different functional category detection preferences. The limited correlation $\left(r^{2}=0.2872\right.$, Figure 4$)$ between the steady-state levels of mRNA and protein abundance is not surprising, because they are individually controlled by their own rates of synthesis and turnover, determined by, for example, gene-specific chromatin structure, sequence content, and binding of accessory proteins. ${ }^{34}$ Alternatively, some observed examples of negative correlation may suggest unexpected biological uncoupling between the expression levels of mRNA and the corresponding proteins.

DNA microarrays are generally considered to have better sensitivity and to provide more comprehensive identification and quantification of transcriptional changes. However, about 67 proteins ( $30 \%$ of the differentially expressed proteins) that were reproducibly identified and quantified by cICAT were not detected by DNA microarray analysis. Many of these orphan proteins were identified with multiple peptide hits; therefore, we are confident of their identification. Most prominent among these proteins are transcription initiation factor IIB (in the nucleic acid metabolism category), microtubule-associated protein RP/EB family member 1, cytochrome P450 51Al, lnnexin 1, and GMP reductase 1 (Table 1). These observations highlight the advantage of the addition of the cICAT strategy to a genomics analysis to reveal subtle changes in the proteome not observed in the transcriptome.

Proteins Differentially Expressed between SC-M1 and TMC-1 Cells. Although the changes in protein and mRNA levels did not show a strong linear correlation, the proteome and transcriptome analysis identified gene products from functionally similar categories. Of particular interest, a significant number of gene products that were up-regulated in TMC-1 cells are involved in cancer metastasis, such as cell-cell adhesion signaling (catenin-120nt, $\alpha$-catenin, $\beta$-catenin, integrin $\alpha 6$, integrin $\beta 4$, RhoG, and IQGAP1), cell metastasis and motility (cytokeratin, myosin, S100 calcium binding protein (S100) family, and vimentin), cell cycle and proliferation (cdc42 and cell division protein kinase 6), tumor immunity and defense (galectin 1 and high mobility group protein 1 ), and protein degradation (cathepsin B, C). The protein expression levels and a comparison of the results of this study with previous reports are shown in Table 3. The different expression levels of these proteins may reflect biological differences leading to the distinct phenotype of the metastatic TMC-1 cell line.

Identification of accurate prognostic factors helps in therapeutic decision-making, in particular in patients with advanced stage cancer. To explore the possibility that the identified proteins may also serve as markers of metastasis for other types of human cancers, we examined the expression of a subset of proteins, $\beta$-catenin, galectin 1 , vimentin, integrin $\alpha 6$, and integrin $\beta 4$ by semiquantitative RT-PCR analysis in various cancer cells with differing invasion potential as assessed by the Boyden chamber assay. Our results showed that higher levels of vimentin and galectin 1 were readily detected in all of the cell lines with demonstrated invasive capability, whereas much lower or insignificant levels of expression were observed in cell lines with low invasiveness (unpublished data, Supplemental Figure 1, Supporting Information). Galectins are a family of structurally related carbohydrate-binding proteins and con- 
Table 3. Summary of Differentially Expressed Proteins Related to Metastasis

\begin{tabular}{|c|c|c|c|c|c|c|c|c|}
\hline \multirow{2}{*}{$\begin{array}{l}\text { gene } \\
\text { name }\end{array}$} & \multirow[b]{2}{*}{ UniProt ID } & \multirow{2}{*}{$\begin{array}{l}\text { protein } \\
\text { name }\end{array}$} & \multirow[b]{2}{*}{ prob. $^{a}$} & \multirow[b]{2}{*}{ molecular function } & \multicolumn{2}{|c|}{ this study } & \multirow{2}{*}{$\begin{array}{l}\text { reproted } \\
\text { expression }_{\text {change }}^{d}\end{array}$} & \multirow[b]{2}{*}{ cancer type $f$} \\
\hline & & & & & $\operatorname{cICAT}^{b}$ & Affymetrix $^{c}$ & & \\
\hline \multicolumn{9}{|c|}{ Cell to Cell Adhesion Signaling } \\
\hline CTNND1 & O60716 & Catenin, 120ctn & 1.00 & $\begin{array}{l}\text { Interacts with cadherin to regulate } \\
\text { cell-adhesion properties }\end{array}$ & 1.78 & 1.21 & Up & $\begin{array}{l}\text { Fibroblasts and Epithelial } \\
\text { Cells (1) }\end{array}$ \\
\hline CTNNA1 & P35221 & Catenin, alpha-1 & 1.00 & $\begin{array}{l}\text { Actin cross linking at adherens } \\
\text { junctions }\end{array}$ & 0.97 & 0.90 & Down & $\begin{array}{l}\text { Lung Carcinomas (2), Prostate } \\
\text { Cancer (3) }\end{array}$ \\
\hline CTNNB1 & P35222 & Catenin, beta & 1.00 & $\begin{array}{l}\text { Modulates of cytoskeletal } \\
\text { dynamics and cell proliferation }\end{array}$ & 5.89 & 1.91 & Down & $\begin{array}{l}\text { Breast Cancer (4), Colorectal } \\
\text { Cancer (5)(6) }\end{array}$ \\
\hline ITGA6 & P23229 & Integrin alpha 6 & 0.99 & $\begin{array}{l}\text { Cell-matrix adhesion,cell- } \\
\text { substrate junction assembly, } \\
\text { cell adhesion }\end{array}$ & 3.34 & 1.81 & Up & Breast Carcinoma Cells (7) \\
\hline ITGB4 & P16144 & Integrin beta 4 & 1.00 & $\begin{array}{l}\text { Cell-matrix adhesion, cell } \\
\text { adhesion }\end{array}$ & 1.96 & 1.80 & Up & Breast Carcinoma Cells (7) \\
\hline PRDX5 & P30044 & Peroxiredoxin 5 & 1.00 & $\begin{array}{l}\text { Contains high antioxidant } \\
\text { efficiency to effect cell } \\
\text { differentiation and apoptosis }\end{array}$ & 1.84 & $\mathrm{NA}^{e}$ & Up & Gastric Cancer (8) \\
\hline PBP & P30086 & $\begin{array}{l}\text { Raf kinase inhibitor } \\
\text { protein }\end{array}$ & 0.98 & $\begin{array}{l}\text { Suppresses metastasis through } \\
\text { decreasing angiogenesis } \\
\text { and vascular invasion }\end{array}$ & 0.48 & 0.62 & Down & Osteosarcoma (9) \\
\hline RHOG & P84097 & $\begin{array}{l}\text { Rho-related } \\
\text { GTP-binding protein } \\
\text { RhoG }\end{array}$ & 1.00 & $\begin{array}{l}\text { Small GTPase-mediated signal } \\
\text { transduction, positive regulation of } \\
\text { cell proliferation, actin cytoskeleton } \\
\text { organization }\end{array}$ & 0.55 & 0.83 & Up & Breast Cancer (10) \\
\hline IQGAP1 & P46940 & $\begin{array}{l}\text { Ras } \\
\text { GTPase-activating-like } \\
\text { protein IQGAP1 }\end{array}$ & 1.00 & $\begin{array}{l}\text { Involves in actin cytoskeleton } \\
\text { assembly and E-cadherin- } \\
\text { mediated cell adhesion }\end{array}$ & 0.35 & 0.19 & Up & Gatric Cancer (11) \\
\hline VCL & P18206 & Vinculin & 1.00 & $\begin{array}{l}\text { Mediates the interactions between } \\
\text { integrins and the actin } \\
\text { cytoskeleton }\end{array}$ & 0.54 & 0.40 & Down & $\begin{array}{l}\text { Basal and Squamous Cell } \\
\text { Tumors (12) }\end{array}$ \\
\hline \multicolumn{9}{|c|}{ Cell Metastasis and Migration } \\
\hline ACTN4 & O43707 & Alpha-actinin 4 & 1.00 & $\begin{array}{l}\text { Anchors actin to a variety of } \\
\text { intracellular structures }\end{array}$ & 1.37 & 1.59 & Up & Colorectal Cancer (13) \\
\hline ANXA1 & P04083 & Annexin I & 1.00 & $\begin{array}{l}\text { Regulates of cell proliferation, } \\
\text { promotes membrane fusion }\end{array}$ & 0.68 & 1.02 & Up/down & $\begin{array}{l}\text { Hepatocellular Carcinoma (14), } \\
\text { Lung Cancer (15), } \\
\text { Head/Neck Cancer (16), Breast } \\
\text { Carcinoma (17) }\end{array}$ \\
\hline KRT17 & Q04695 & Cytoskeletal 17 & 1.00 & $\begin{array}{l}\text { Marker of basal cell differentiation } \\
\text { in complex epithelia }\end{array}$ & 0.03 & $\mathrm{NA}^{e}$ & Up & Breast Cancer (18) \\
\hline KRT5 & P13647 & Cytoskeletal 5 & 1.00 & $\begin{array}{l}\text { Epidermis development, } \\
\text { cytoskeleton organization and } \\
\text { biogenesis, cellular } \\
\text { morphogenesis }\end{array}$ & 3.43 & $\mathrm{NA}^{e}$ & Up & Breast Cancer (18) \\
\hline TPM3 & Q8NAG3 & Tropomyosin 3 & 1.00 & Stabilizes of actin filaments & 0.48 & $\mathrm{NA}^{e}$ & Down & $\begin{array}{l}\text { Prostate Cancer (19), Breast } \\
\text { Cancer (20) }\end{array}$ \\
\hline TPM4 & P67936 & Tropomyosin 4 & 1.00 & $\begin{array}{l}\text { Binds to actin filaments in muscle } \\
\text { and nonmuscle cells }\end{array}$ & 0.61 & 0.82 & Up/down & Lung Cancer (21) \\
\hline VIM & P08670 & Vimentin & 0.99 & $\begin{array}{l}\text { Intermediate filament-based } \\
\text { process in mesenchymal cells } \\
\text { related to migration }\end{array}$ & 25.67 & $\mathrm{NA}^{e}$ & Up & $\begin{array}{l}\text { Breast Carcinoma (17), } \\
\text { Hepatocellular Carcinoma (22), } \\
\text { Propstate Cancer (23) }\end{array}$ \\
\hline S100A11 & P31949 & $\begin{array}{l}\text { S100 calcium-binding } \\
\text { protein All }\end{array}$ & 1.00 & $\begin{array}{l}\text { Actin filament bundle formation } \\
\text { and cell motility }\end{array}$ & 1.34 & $\mathrm{NA}^{e}$ & Up & Hepatocellular Carcinoma (14) \\
\hline COMT & P21964 & $\begin{array}{l}\text { Catechol } \\
O \text {-methyltransferase }\end{array}$ & 1.00 & $\begin{array}{l}\text { Involved in cancer progression and } \\
\text { lymph node metastasis }\end{array}$ & 1.35 & 0.81 & Up & Breast Cancer (24) \\
\hline \multicolumn{9}{|c|}{ Cell Cycle and Cell Proliferation Regulation } \\
\hline SKP1A & P63208 & $\begin{array}{l}\text { S-phase } \\
\text { kinase-associated } \\
\text { protein 1A }\end{array}$ & 0.97 & $\begin{array}{l}\text { Mediates the ubiquitination of } \\
\text { proteins involved in cell cycle } \\
\text { progression, signal transduction } \\
\text { and transcription }\end{array}$ & 0.35 & 0.80 & Up & $\begin{array}{l}\text { Colorectal Carcinoma (25), } \\
\text { Hepatocellular Carcinoma (26) }\end{array}$ \\
\hline CDC42 & P60953 & $\begin{array}{l}\text { Cell division control } \\
\text { protein } 42 \text { homologue }\end{array}$ & 1.00 & $\begin{array}{l}\text { Regulates cadherin-mediated } \\
\text { cell-cell adhesion }\end{array}$ & 0.67 & 0.88 & Up & Breast Cancer (27) \\
\hline CDK6 & Q00534 & Cell division kinase 6 & 1.00 & $\begin{array}{l}\text { Related to cell proliferation, tumor } \\
\text { heterogeneity, invasion and } \\
\text { metastasis }\end{array}$ & 1.72 & $\mathrm{NA}^{e}$ & Up & $\begin{array}{l}\text { Retinoblastoma (28)Immunity } \\
\text { and Defense }\end{array}$ \\
\hline HMGB1 & P09429 & $\begin{array}{l}\text { High mobility group } \\
\text { protein } 1\end{array}$ & 1.00 & $\begin{array}{l}\text { Immunity and Defense } \\
\text { Interacts with transcription factors } \\
\text { and regulates of transcription } \\
\text { related to tumor growth and } \\
\text { invasion }\end{array}$ & 1.92 & 1.24 & Up & $\begin{array}{l}\text { Gastrointestinal Stromal } \\
\text { Tumors (29) }\end{array}$ \\
\hline CD44 & Q16208 & CD44 & 0.96 & $\begin{array}{l}\text { Mediates cell-cell and cell-matrix } \\
\text { interactions through high affinity for } \\
\text { hyaluronic acid }\end{array}$ & 1.01 & 0.58 & Up & $\begin{array}{l}\text { Melanoma (30), Gastric } \\
\text { Carcinoma (31) }\end{array}$ \\
\hline LGALS1 & P09382 & Galectin-1 & 1.00 & $\begin{array}{l}\text { Promotes cancer cell invasion and } \\
\text { metastasis }\end{array}$ & 2.33 & 0.90 & Up & $\begin{array}{l}\text { Breast Carcinoma (32), } \\
\text { Astrocytoma, Melanoma, } \\
\text { Prostate, Colon, Bladder, } \\
\text { Ovary (33) }\end{array}$ \\
\hline HSPD1 & P10809 & Heat shock protein 60 & 1.00 & $\begin{array}{l}\text { Facilitates the correct folding of } \\
\text { imported proteins }\end{array}$ & 1.5 & 1.12 & Down & Lung Cancer (15) \\
\hline HSPCB & P08238 & $\begin{array}{l}\text { Heat shock protein } 90 \\
\text { beta }\end{array}$ & 1.00 & $\begin{array}{l}\text { Promotes the maturation of MMP2 } \\
\text { involved in invasive cancer }\end{array}$ & 0.68 & 0.87 & Up & Murine Fibrosarcoma (34) \\
\hline HLAA & P10892 & MHC class I antigen & 1.00 & $\begin{array}{l}\text { Inhibits evasion of the immune } \\
\text { system and enhances tumor } \\
\text { growth }\end{array}$ & 0.64 & $\mathrm{NA}^{e}$ & Up & Gastric Cancer (35) \\
\hline
\end{tabular}


Table 3. (Continued)

\begin{tabular}{|c|c|c|c|c|c|c|c|c|}
\hline \multirow{2}{*}{$\begin{array}{l}\text { gene } \\
\text { name }\end{array}$} & \multirow[b]{2}{*}{ UniProt ID } & \multirow{2}{*}{$\begin{array}{l}\text { protein } \\
\text { name }\end{array}$} & \multirow[b]{2}{*}{ prob. $^{a}$} & \multirow[b]{2}{*}{ molecular function } & \multicolumn{2}{|c|}{ this study } & \multirow{2}{*}{$\begin{array}{l}\text { reproted } \\
\text { expression }^{\text {change }}{ }^{d}\end{array}$} & \multirow[b]{2}{*}{ cancer type $^{f}$} \\
\hline & & & & & $\operatorname{cICAT}^{b}$ & Affymetrix $^{c}$ & & \\
\hline \multicolumn{9}{|c|}{ Protein Degradation } \\
\hline CTSB & P07858 & Cathepsin B & 1.00 & $\begin{array}{l}\text { Protein targeting, proteolysis and } \\
\text { peptidolysis }\end{array}$ & 1.61 & 2.16 & Up & $\begin{array}{l}\text { Melanoma (30), Breast Cancer } \\
\text { (4), Lung cancer (31) }\end{array}$ \\
\hline CTSC & P53634 & Cathepsin C & 1.00 & $\begin{array}{l}\text { Intracellular protein degradation } \\
\text { and turnover }\end{array}$ & 1.62 & 0.28 & Up & Nonsmall Cell Lung Cancer (36) \\
\hline CCT2 & P78371 & $\begin{array}{l}\text { T-complex protein beta } \\
\text { subunit }\end{array}$ & 1.00 & $\begin{array}{l}\text { Related to p53 and activates DNA } \\
\text { damage checkpoints }\end{array}$ & 1.23 & $\mathrm{NA}^{e}$ & Down & Breast Carcinoma (17) \\
\hline
\end{tabular}

${ }^{a}$ ProteinProphet Score. ${ }^{b}$ The fold change in the protein ratio of TMC-1/SC-M1 cells. ${ }^{c}$ The fold change in the mRNA ratio of TMC-1/SC-M1 cells. ${ }^{d}$ Literature report(s) of change in protein level in metastatic specimens. ${ }^{e}$ NA, not avaiable. ${ }^{f}$ References were provided as Supporting Information.

A

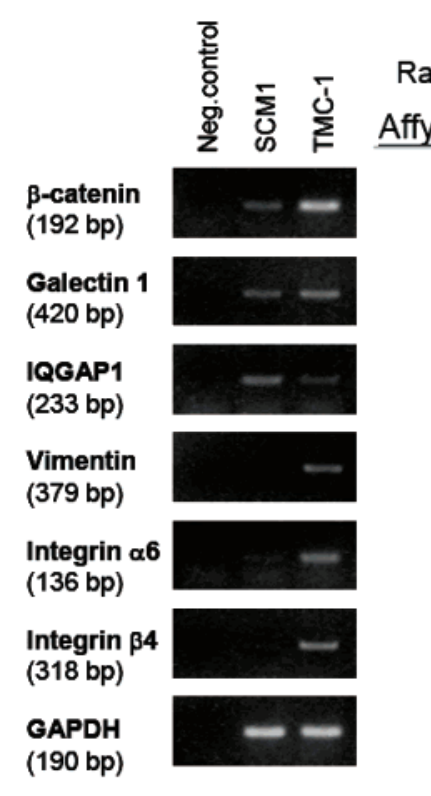

B

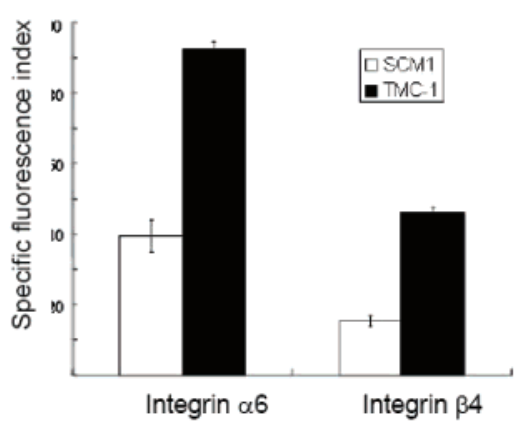

N.A $\quad 25.67$

1.81

3.34

1.92

0.89

5.89

2.33

0.35

C
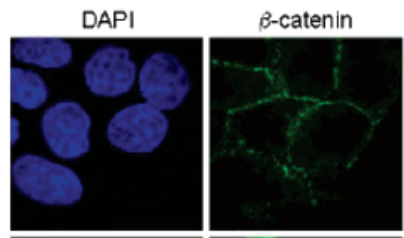

0.82
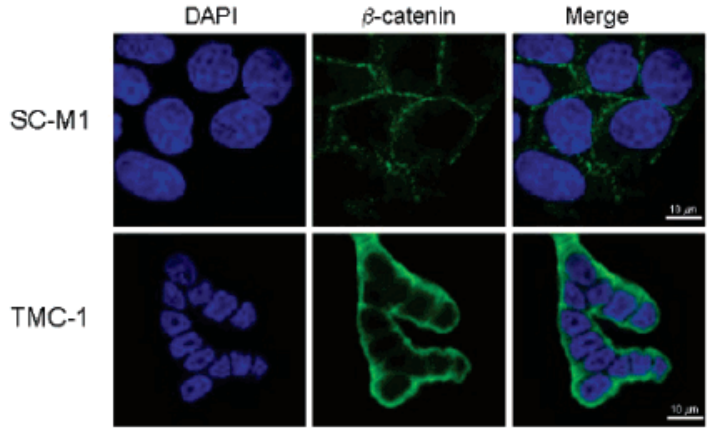

Figure 5. Expression of candidate genes in SC-M1 and TMC-1cell lines. (A) RT-PCR analysis of $\beta$-catenin, galectin 1, vimentin, integrin $\alpha 6$, and integrin $\beta 4$ transcripts. The TMC-1/SC-M1 cell expression ratios obtained by Affymetrix DNA microarray and clCAT analysis are also shown for comparison. (B) Cell surface expression of integrins 6 and 4 as measured by flow cytometry. The specific fluorescence index was calculated as the ratio of the mean fluorescence values obtained with the specific $A b$ and the isotype control Ab. (C) The immunocytostaining images of $\beta$-catenin in SC-M1 and TMC-1 cells obtained by confocal laser microscopy.

tribute to tumor transformation, cell cycle regulation, apoptosis, cell adhesion, migration, and inflammation. ${ }^{9,10}$ Their expression correlates with tumor aggressiveness and acquisition of a metastatic phenotype in different tumor types. ${ }^{35}$ Vimentin is a cytoskeletal protein that is often expressed when epithelial cells are stimulated to proliferate by growth factors. ${ }^{36}$ In cancer, vimentin expression is associated with a dedifferentiated malignant phenotype, increased motility and invasiveness, and poor clinical prognosis. ${ }^{36}$ The significant overexpression of galectin 1 and vimentin we observed in TMC-1 cells is consistent with the reported correlation between cellular adhesion and tumor aggregation ${ }^{37}$ and may be possible markers for metastasis. ${ }^{38}$

Although expression level changes in all of the proteins listed in Table 3 have been shown to be closely related to cancer metastasis, to the best of our knowledge, most have not been linked to gastric cancer. Previous studies have shown that the members of S100 family, S100A4, S100A6, and S100A11, are involved in cell motility, proliferation, differentiation, and cancer cell progression in breast cancer, colorectal cancer, ${ }^{39}$ gall bladder cancer, and hepatocellular carcinomas. ${ }^{40} \mathrm{We}$ also found overexpression of the S100A11 protein in TMC-1 cells. Annexin I is reportedly up-regulated in highly metastatic lung carcinoma cell lines ${ }^{41}$ and down-regulated in a nonmetastatic type of breast cancer. ${ }^{42}$ Increased expression of S100A11 may be correlated with observed down-regulation of Annexin I, the binding target of S100A11.

In addition, cathepsin $\mathrm{B}$ and cathepsin $\mathrm{C}$ were up-regulated in metastatic TMC-1 cells compared to the nonmetastatic SCM1 cells. Elevated expression of these lysosomal proteases, cathepsins B, L, and D, and diminished levels of their inhibitors have been observed in breast and prostate cancer, especially in aggressive cancer cells. ${ }^{43}$ Evidence suggests that capthepsin $\mathrm{B}$ has an important function in matrix degradation and invasion, facilitating the growth capability of invasive carcinomas through loss of calcium-dependent cell-cell adhesion and proteolysis of E-cadherin, which is a tumor suppressor and effector of adherens junction function. ${ }^{44}$ Our results suggest that cathepsin $\mathrm{C}$ may also be involved in the metastasis of gastric cancer cells. 

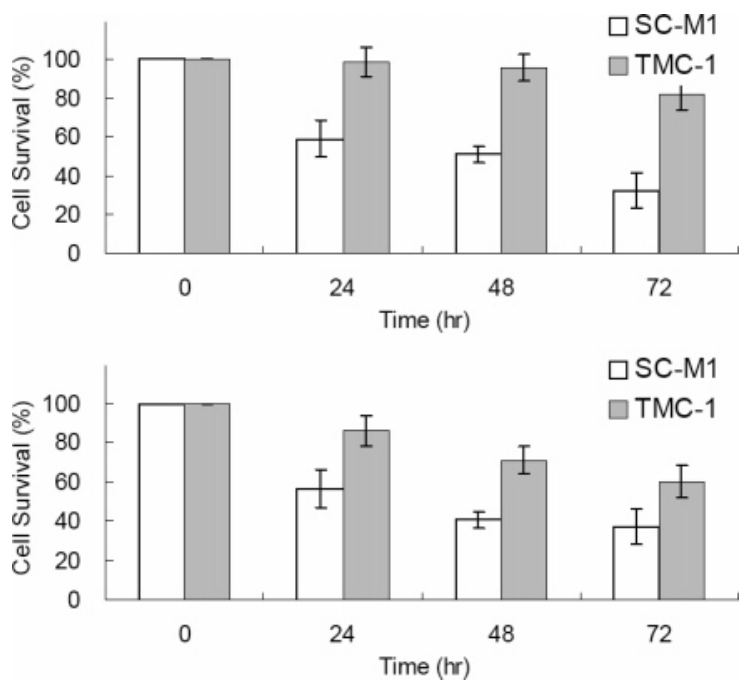

Figure 6. Inhibition of cell viability of the SCM- 1 and TMC- 1 cell lines by 5 -fluorouracil. The cell lines were incubated with $(A) 10$ $\mu \mathrm{M}$ or (B) $50 \mu \mathrm{M} 5$-fluorouracil for 24,48 , and $72 \mathrm{~h}$. Cells were then assayed by Alamar Blue dye uptake.

Metastasis-Perturbed Pathway. Furthermore, the differentially expressed proteins identified in the current comparative approach enabled us to study their correlations in multiple cellular pathways. Using the pathway analysis tools available at the BioCarta, KEGG, PANTHER, and PathwayAssist, we found differences in expression of proteins in the pathways related to cytoskeleton rearrangement, cell-cell adhesion, and cellECM attachment between the metastatic TMC-1 and nonmetastatic SC-M1 cells. Figure 7 depicts a summary of these proteins and pathways, including the integrin signaling pathway, the
Rho cell motility signaling pathway, and pathways associated with cell-cell adhesion and adherens junctions.

Recently, increasing evidence suggests that epithelial-mesenchymal transitions (EMT) play a specific role in the migration of cells from primary tumors into the circulation. ${ }^{45}$ The defining event for the epithelial-mesenchymal transition is disruption of E-cadherin-mediated cell-cell adhesion, which results in loss of epithelial morphology and acquisition of a motile, mesenchymal phenotype. ${ }^{46}$ Cancer cells that undergo the EMT, therefore, lose their ability to adhere to their neighboring cells, resulting in weakened cell-cell adhesion and subsequent tumor cell migration. Much evidence indicates that loss or reduction of E-cadherin-mediated adhesion, thus, allowing cell migration, is required for the metastatic conversion of carcinomas. Cadherins are calcium-dependent adhesion molecules responsible for the establishment of tight cell-cell contact. Anchorage to the actin cytoskeleton occurs through the binding of the cytoplasmic domain of cadherins to $\beta$-catenin or $\gamma$-catenin, which concurrently binds to $\alpha$-catenin. ${ }^{47,48} \beta$-Catenin plays a central role in linking the membrane-bound E-cadherin to the cytoskeleton, and the interaction of $\beta$-catenin and IQGAP1 negatively regulates E-cadherin-mediated cell-cell adhesion. ${ }^{49}$ Fukata et al. have proposed that $\beta$-catenin exists in a dynamic equilibrium between the E-cadherin- $\beta$-catenin$\alpha$-catenin complex and the E-cadherin- $\beta$-catenin-IQGAP1 complex at sites of cell-cell contact. Weakening cell-cell adhesion shifts the balance of $\beta$-catenin from the cadherinbound pool to the cytoplasmic pool. In this study, immunocytostaining showed more cytoplasmic localization of $\beta$-catenin in the metastatic TMC-1 cells (Figure 5). A newly discovered $\beta$-catenin interaction partner, 14-3-3 $\zeta, 50$ enhances the transcription of $\beta$-catenin target promoters, leading to elevated

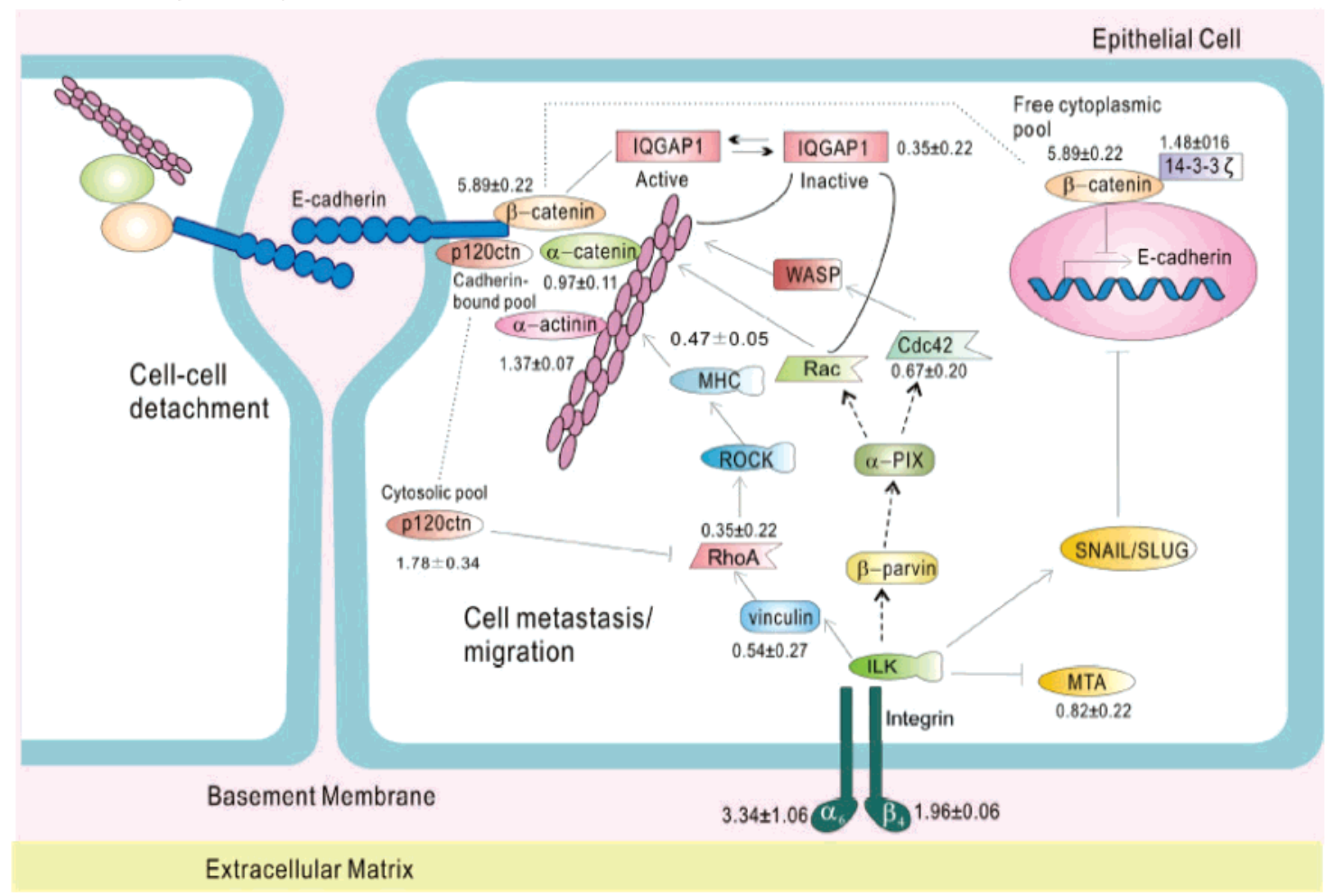

Figure 7. Summary of differentially expressed proteins in multiple pathways involved in cell-cell adhesion and cell-ECM interactions. The fold changes and standard deviation of the cICAT TMC-1/SC-M1 cell expression ratios are indicated. The solid lines indicate pathways derived from the BioCarta, KEGG, and PANTHER databases, and dashed lines indicate those found in the literature (using PathwayAssist). 
steady-state levels of $\beta$-catenin in the cytoplasm. The accumulation of free cytosolic $\beta$-catenin has been reported to lead to translocation of $\beta$-catenin to the nucleus, which results in sustained transcriptional activation of oncogenes and causes tumor growth..$^{51}$ Although we did not observe $\beta$-catenin in the nucleus, up-regulation of both 14-3-3 $\xi$ and $\beta$-catenin in TMC-1 cells was observed, which suggests activation of the Wnt signaling pathway..$^{50}$

In addition, anchoring junction dynamics can be regulated by intricate interactions of the cadherin-catenin complex with Cdc42, a member of the Rho GTPase subfamily known for its role in regulating cell division. ${ }^{22} \mathrm{Racl}$ and Cdc42 might also regulate the cleavage and endocytosis of E-cadherin, thereby modifying E-cadherin-mediated cell-cell adhesion. Meanwhile, Cdc42 and Racl also regulate cell-cell adhesion through IQGAP1 by the interaction of IQGAP1 with $\beta$-catenin, leading to stabilization of the E-cadherin-catenin complex. ${ }^{53,54}$ The decreased levels of IQGAP1 and Cdc42 we observed seem to imply that the Rac1-Cdc42-IQGAP1 complex is involved in the weak intercellular adhesion of TMC-1 cells.

The dysregulation of ECM adhesion, which is mediated mainly by integrins through GTPases, is another crucial determinant for tumor progression. ${ }^{55} \mathrm{E}$-cadherin-mediated adhesion may also be regulated by integrin $\alpha_{6} \beta_{4}$ signaling through phosphatidylinositol 3 kinase to Rac, or alternatively, the transcription of E-cadherin can be suppressed through integrinlinked kinase (ILK) by up-regulating the transcriptional suppression of E-cadherin by repressors SNAL/SLUG ${ }^{55}$ In addition, the p120ctn in the cytosol and in the cadherin-based complex provides one mechanism for communication between cadherin-mediated cell-cell junctions and the motile machinery of cells ${ }^{56}$ The observed up-regulation of p120ctn and downregulation of RhoA suggest that p120ctn may negatively modulate RhoA to affect other downstream effectors. Active RhoA increases the stability of actin-based focal adhesions. Therefore, the decreased level of RhoA and its downstream effectors, vinculin and myosin heavy/light chain (MHC), that we observed in the cICAT analysis of TMC-1 cells also implies a weakened capability to adhere to the ECM.

In general, advanced gastric cancer is refractory to chemotherapy, leading to poor prognosis. To search for novel targets for screening and therapeutic intervention, it is increasingly important to recognize that discovery of disease-perturbed pathways is more fruitful than identification of individual gene products. ${ }^{57}$ In summary, our results that both the cell-cell adhesion and the cell-ECM attachment pathways are disrupted provide identification of several differentially expressed pathways. Their associated proteins may be potential biomarkers of metastatic gastric tumor cells and of invasiveness. The regulation of cell-cell adhesion or dissociation is extremely complicated, and the plasticity the cells exhibit likely requires real-time control by transcriptional networks. Further investigation on the temporal profiling of these complex signaling cascades will provide insight into the invasive behavior and the metastatic potential of cancer cells.

Abbreviations: cICAT, cleavable isotope-coded affinity tag; TCEP, Tris-(2-carboxyethyl)phosphine; SCX, strong cation exchange; DAPI, 4',6-diamidino-2-phenylindole-dihydrochloride; ECM, extracellular matrix.

Acknowledgment. The authors acknowledge financial support from Academia Sinica and National Science Council in Taiwan. We are also grateful to Prof. Ruedi Aebersold, Prof.
David R. Goodlett, and Drs. Eugene C. Yi, and Hookeun Lee at Institute for Systems Biology, Seattle WA, for helpful advice of cICAT analysis.

Supporting Information Available: Figure showing the semiquantitative analysis of galectin 1, vimentin, IQGAP1, and $\beta$-catenin transcripts in different cancer cell lines, including breast, colon, ovary, bladder, and lung cancer; tables showing the protein identification summaries of TMC-1 and SC-M1 cells and of the cICAT analysis. This material is available free of charge via the Internet at http://pubs.acs.org.

\section{References}

(1) Parkin, D. M.; Pisani, P.; Ferlay, J. Estimates of the worldwide incidence of 25 major cancers in 1990. Int. J. Cancer 1999, 80, 827-41.

(2) Stadtlander, C. T.; Waterbor, J. W. Molecular epidemiology, pathogenesis and prevention of gastric cancer. Carcinogenesis 1999, 20, 2195-208.

(3) Peddanna, N.; Holt, S.; Verma, R. S. Genetics of gastric cancer. Anticancer Res. 1995, 15, 2055-64.

(4) Hippo, Y.; Taniguchi, H.; Tsutsumi, S.; et al. Global gene expression analysis of gastric cancer by oligonucleotide microarrays. Cancer Res. 2002, 62, 233-40.

(5) Cai, Z.; Chiu, J. F.; He, Q. Y. Application of proteomics in the study of tumor metastasis. Genomics, Proteomics Bioinf. 2004, 2, 152-66.

(6) Wang, W.; Goswami, S.; Sahai, E.; Wyckoff, J. B.; Segall, J. E.; Condeelis, J. S. Tumor cells caught in the act of invading,, their strategy for enhanced cell motility. Trends Cell Biol. 2005, 15, $138-45$.

(7) Lin, B.; White, J. T.; Lu, W.; et al. Evidence for the presence of disease-perturbed networks in prostate cancer cells by genomic and proteomic analyses: a systems approach to disease. Cancer Res. 2005, 65, 3081-91.

(8) Sinha, P.; Poland, J.; Schnolzer, M.; Celis, J. E.; Lage, H. Characterization of the differential protein expression associated with thermoresistance in human gastric carcinoma cell lines. Electrophoresis 2001, 22, 2990-3000.

(9) Choi, Y. R.; Kim, H.; Kang, H. J.; et al. Overexpression of high mobility group box 1 in gastrointestinal stromal tumors with KIT mutation. Cancer Res. 2003, 63, 2188-93.

(10) Chen, J.; Kahne, T.; Rocken, C.; et al. Proteome analysis of gastric cancer metastasis by two-dimensional gel electrophoresis and matrix assisted laser desorption/ionization-mass spectrometry for identification of metastasis-related proteins. J. Proteome Res. 2004, 3, 1009-16.

(11) He, Q. Y.; Cheung, Y. H.; Leung, S. Y.; Yuen, S. T.; Chu, K. M.; Chiu, J. F. Diverse proteomic alterations in gastric adenocarcinoma. Proteomics 2004, 4, 3276-87.

(12) Das, S.; Sierra, J. C.; Soman, K. V.; et al. Differential protein expression profiles of gastric epithelial cells following Helicobacter pylori infection using ProteinChips. J. Proteome Res. 2005, 4, 92030.

(13) Juan, H. F.; Chen, J. H.; Hsu, W. T.; et al. Identification of tumorassociated plasma biomarkers using proteomic techniques: from mouse to human. Proteomics 2004, 4, 2766-75.

(14) Washburn, M. P.; Wolters, D.; Yates, J. R., III. Large-scale analysis of the yeast proteome by multidimensional protein identification technology. Nat. Biotechnol. 2001, 19, 242-7.

(15) Washburn, M. P.; Ulaszek, R.; Deciu, C.; Schieltz, D. M.; Yates, J. R., III. Analysis of quantitative proteomic data generated via multidimensional protein identification technology. Anal. Chem. 2002, 74, 1650-7.

(16) Aebersold, R.; Mann, M. Mass spectrometry-based proteomics. Nature 2003, 422, 198-207.

(17) Gygi, S. P.; Rist, B.; Gerber, S. A.; Turecek, F.; Gelb, M. H.; Aebersold, R. Quantitative analysis of complex protein mixtures using isotope-coded affinity tags. Nat. Biotechnol. 1999, 17, 9949 .

(18) Li, J.; Steen, H.; Gygi, S. P. Protein profiling with cleavable isotopecoded affinity tag (cICAT) reagents: the yeast salinity stress response. Mol. Cell. Proteomics 2003, 2, 1198-204.

(19) Li, C.; Hong, Y.; Tan, Y. X.; et al. Accurate qualitative and quantitative proteomic analysis of clinical hepatocellular carcinoma using laser capture microdissection coupled with isotopecoded affinity tag and two-dimensional liquid chromatography mass spectrometry. Mol. Cell. Proteomics 2004, 3, 399-409. 
(20) Tzeng, C. C.; Meng, C. L.; Jin, L.; Hsieh, H. F. Cytogenetic studies of gastric adenocarcinoma. Cancer Genet. Cytogenet. 1991, 55, $67-71$

(21) Shyu, R. Y.; Jiang, S. Y.; Jong, Y. J.; et al. Establishment and characterization of a human gastric carcinoma cell line TMC-1. Cells Tissues Organs 2004, 177, 37-46.

(22) Keller, A.; Nesvizhskii, AI.; Kolker, E.; Aebersold, R. Empirical statistical model to estimate the accuracy of peptide identifications made by MS/MS and database search. Anal. Chem. 2002, 74, 5383-92.

(23) Nesvizhskii, A. I.; Keller, A.; Kolker, E.; Aebersold, R. A statistical model for identifying proteins by tandem mass spectrometry. Anal. Chem. 2003, 75, 4646-58.

(24) Li, X. J.; Zhang, H.; Ranish, J. A.; Aebersold, R. Automated statistical analysis of protein abundance ratios from data generated by stable-isotope dilution and tandem mass spectrometry. Anal. Chem. 2003, 75, 6648-57.

(25) Kim, J. M.; Sohn, H. Y.; Yoon, S. Y.; et al. Identification of gastric cancer-related genes using a cDNA microarray containing novel expressed sequence tags expressed in gastric cancer cells. Clin. Cancer Res. 2005, 11, 473-82.

(26) Wechsler-Reya, R. J.; Elliott, K. J.; Prendergast, G. C. A role for the putative tumor suppressor Binl in muscle cell differentiation. Mol. Cell. Biol. 1998, 18, 566-75.

(27) Su, M. W.; Dorocicz, I.; Dragowska, W. H.; et al. Aberrant expression of T-plastin in Sezary cells. Cancer Res. 2003, 63 7122-7.

(28) Gerk, P. M.; Vore, M. Regulation of expression of the multidrug resistance-associated protein 2 (MRP2) and its role in drug disposition. J. Pharmacol. Exp. Ther. 2002, 302, 407-15.

(29) Reiher, F. K.; Volpert, O. V.; Jimenez, B.; et al. Inhibition of tumor growth by systemic treatment with thrombospondin-1 peptide mimetics. Int. J. Cancer 2002, 98, 682-9.

(30) Nishizuka, S.; Charboneau, L.; Young, L.; et al. Proteomic profiling of the NCI-60 cancer cell lines using new high-density reversephase lysate microarrays. Proc. Natl. Acad. Sci. U.S.A. 2003, 100, 14229-34.

(31) Mulholland, D. J.; Dedhar, S.; Coetzee, G. A.; Nelson, C. C. Interaction of nuclear receptors with the Wnt/beta-catenin/Tcf signaling axis: Wnt you like to know? Endocr. Rev. 2005, 26, 898915.

(32) Feng, Y.; Lee, N.; Fearon, E. R. TIP49 regulates beta-cateninmediated neoplastic transformation and $\mathrm{T}$-cell factor target gene induction via effects on chromatin remodeling. Cancer Res. 2003, 63, 8726-34.

(33) Oliver, L.; Cordel, S.; Barbieux, I.; et al. Resistance to apoptosis is increased during metastatic dissemination of colon cancer. Clin. Exp. Metastasis 2002, 19, 175-80.

(34) Dechering, K. J. The transcriptome's drugable frequenters. Drug Discovery Today 2005, 10, 857-64.

(35) Rabinovich, G, A. Galectin-1 as a potential cancer target. Br. J. Cancer 2005, 92, 1188-92.

(36) Yoon, W. H.; Song, I. S.; Lee, B. H.; et al. Differential regulation of vimentin mRNA by 12-O-tetradecanoylphorbol 13-acetate and all-trans-retinoic acid correlates with motility of Hep 3B human hepatocellular carcinoma cells. Cancer Lett. 2004, 203, 99-105.

(37) Kasamatsu, A.; Uzawa, K.; Nakashima, D.; et al. Galectin-9 as a regulator of cellular adhesion in human oral squamous cell carcinoma cell lines. Int. J. Mol. Med. 2005, 16, 269-73.

(38) Irie, A.; Yamauchi, A.; Kontani, K.; et al. Galectin-9 as a prognostic factor with antimetastatic potential in breast cancer. Clin. Cancer Res. 2005, 11, 2962-8.
(39) Komatsu, K.; Kobune-Fujiwara, Y.; Andoh, A.; et al. Increased expression of S100A6 at the invading fronts of the primary lesion and liver metastasis in patients with colorectal adenocarcinoma. Br. J. Cancer 2000, 83, 769-74.

(40) Cui, J. F.; Liu, Y. K.; Pan, B. S.; et al. Differential proteomic analysis of human hepatocellular carcinoma cell line metastasis-associated proteins. J. Cancer Res. Clin. Oncol. 2004, 130, 615-22.

(41) Jiang, D.; Ying, W.; Lu, Y.; et al. Identification of metastasisassociated proteins by proteomic analysis and functional exploration of interleukin-18 in metastasis. Proteomics 2003, 3, 72437.

(42) Kreunin, P.; Urquidi, V.; Lubman, D. M.; Goodison, S. Identification of metastasis-associated proteins in a human tumor metastasis model using the mass-mapping technique. Proteomics 2004, 4, 2754-65.

(43) Nomura, T.; Katunuma, N. Involvement of cathepsins in the invasion, metastasis and proliferation of cancer cells. J. Med. Invest. 2005, 52, 1-9.

(44) Jawhari, A. U.; Noda, M.; Farthing, M. J.; Pignatelli, M. Abnormal expression and function of the E-cadherin-catenin complex in gastric carcinoma cell lines. Br. J. Cancer 1999, 80, 322-30.

(45) Huber, M. A.; Kraut, N.; Beug, H. Molecular requirements for epithelial-mesenchymal transition during tumor progression. Curr. Opin. Cell Biol. 2005, 17, 548-58.

(46) Rosivatz, E.; Becker, I.; Specht, K.; et al. Differential expression of the epithelial-mesenchymal transition regulators snail, SIP1 and twist in gastric cancer. Am. J. Pathol. 2002, 161, 1881-91.

(47) Beavon, I. R. The E-cadherin-catenin complex in tumour metastasis: structure, function and regulation. Eur. J. Cancer 2000 , $36,1607-20$

(48) Fukata, M.; Nakagawa, M.; Itoh, N.; et al. Involvement of IQGAP1, an effector of Rac1 and Cdc42 GTPases, in cell-cell dissociation during cell scattering. Mol. Cell. Biol. 2001, 21, 2165-83.

(49) Fukata, M.; Kaibuchi, K. Rho-family GTPases in cadherinmediated cell-cell adhesion. Nat. Rev. Mol. Cell Biol. 2001, 2 887-97.

(50) Tian, Q.; Feetham, M. C.; Tao, W. A.; et al. Proteomic analysis identifies that 14-3-3zeta interacts with beta-catenin and facilitates its activation by Akt. Proc. Natl. Acad. Sci. U.S.A. 2004, 101, 15370-5.

(51) Sparks, A. B.; Morin, P. J.; Vogelstein, B.; Kinzler, K. W. Mutational analysis of the APC/beta-catenin/Tcf pathway in colorectal cancer. Cancer Res. 1998, 58, 1130-4.

(52) Lui, W. Y.; Mruk, D. D.; Cheng, C. Y. Interactions among IQGAP1, Cdc42, and the cadherin/catenin protein complex regulate Sertoli-germ cell adherens junction dynamics in the testis. J. Cell. Physiol. 2005, 202, 49-66.

(53) Kuroda, S.; Fukata, M.; Nakagawa, M.; et al. Role of IQGAP1, a target of the small GTPases Cdc42 and Rac1, in regulation of E-cadherin-mediated cell-cell adhesion. Science 1998, 281, 8325 .

(54) Fukata, M.; Kuroda, S.; Nakagawa, M.; et al. Cdc42 and Rac1 regulate the interaction of IQGAP1 with beta-catenin. J. Biol Chem. 1999, 274, 26044-50.

(55) Guo, W.; Giancotti, F. G. Integrin signalling during tumour progression. Nat. Rev. Mol. Cell Biol. 2004, 5, 816-26.

(56) Noren, N. K.; Liu, B. P.; Burridge, K.; Kreft, B. p120 catenin regulates the actin cytoskeleton via Rho family GTPases. J. Cell. Biol. 2000, 150, 567-80.

(57) Kramer, R.; Cohen, D. Functional genomics to new drug targets. Nat. Rev. Drug Discovery 2004, 3, 965-72.

PR060212G 